Mathematical Models and Methods in Applied Sciences

(c) World Scientific Publishing Company

\title{
ON THE WELL-POSEDNESS OF A COUPLED ONE-DIMENSIONAL BIOLOGICAL-PHYSICAL MODEL FOR THE UPPER OCEAN
}

\author{
BLAISE FAUGERAS \\ MAPLY, Centre de mathématique INSA de Lyon, \\ Bat. Léonard de Vinci, 21 avenue Jean Capelle, \\ 69621, Villeurbanne Cedex, France. \\ faugeras@laninsa.insa-lyon.fr
}

\begin{abstract}
This paper introduces a one-dimensional NPZD-model developed to simulate biological activity in a turbulent ocean water column. The model consists of a system of coupled semilinear parabolic equations. An initial-boundary value problem is formulated and the existence of a unique positive weak solution to it is proved. The existence result is derived using a variational formulation, an approximate model and a fixed-point method. It is shown that the qualitative analysis performed still applies if different parameterizations of several biological processes found in the biogeochemical modeling literature are used.

Keywords: Marine ecosystem model; semilinear parabolic system; variational formulation; positivity.
\end{abstract}

AMS Subject Classification: 86A05, 92F05, 35K45, 35K50, 35K57, 35R05

\section{Introduction}

Within the scope of global climate studies, authors carrying out modeling research use marine ecosystem models with increasing degrees of complexity. Complexity in such models can arise from the number of biological compartments, or state variables, which are taken into account, as well as from the parameterizations used to model interactions between these compartments. The number of variables can vary from one to more than ten. At least two variables, nutrients $(N)$ and phytoplankton $(P)$ are necessary to model primary production, that is to say the transformation of mineral nutrients into primitive biotic material using external energy, provided by the sun (Taylor et al. ${ }^{28}$ ). However, in order to study the ocean carbon cycle, the main biological processes which have to be understood and estimated are primary production, but also the export of organic matter from the surface to deep ocean layers and organic matter remineralization. The simpliest model able to represent all these processes contains four variables, nutrients $(N)$, phytoplankton $(P)$, zooplankton $(Z)$ and detritus $(D)$. This type of model is termed the NPZD-model and 
different variants of it are used in numerous studies. All these models are similar from a structural point of view but authors use different parameterizations to model fluxes between biological compartments.

Complexity can also arise from the spatial resolution of the physical dynamics to which biological variables are submitted. Many model set-ups are zero-dimensional and biological variables correspond to ocean mixed-layer values (e.g., Fasham et al. ${ }^{10}$, Steele and Henderson ${ }^{27}$, Spitz et al. ${ }^{26}$, Fennel et al. $\left.{ }^{13}\right)$. Others are onedimensional, considering that the ocean, in some particular places, can be modeled with a good approximation by a turbulent water column (e.g., Prunet et al. ${ }^{25}$, Doney et al. ${ }^{8}$, Lévy et al. ${ }^{16}$, Mémery et al. ${ }^{22}$ ). Finally, in some studies, the biological model is integrated in a three-dimensional circulation model (e.g., Fasham et al. ${ }^{11}$, Moisan et al. ${ }^{23}$, Lévy et al. ${ }^{17}$, Carmillet et al. ${ }^{6}$ ). The question which has motivated this work is: are all these models well-posed? Of course, it seems difficult to study all of them and in this work we concentrate first on a one-dimensional NPZD-model and then discuss the possible generalization of our result. The three-dimensional version of the model we consider is proposed in Lévy et al. ${ }^{17}$, and a one-dimensional version of a similar model, containing six biological variables, is used by Faugeras et al. ${ }^{12}$ to assimilate data from the JGOFS-DYFAMED time-series station in the North-Western Mediterranean Sea.

Mathematically, the biological model under consideration is a system of coupled parabolic semilinear equations to which initial and boundary conditions are added. Under certain hypotheses, this general type of initial-boundary value problem can be transformed to an abstract Cauchy problem and studied using the theory of semigroups (following Chapter 6 of the book by Pazy ${ }^{24}$ for example). In their paper Boushaba et al. ${ }^{4}$ used results on semigroups to provide a mathematical analysis of a model describing the evolution of a single variable phytoplankton. Although the model they considered is three-dimensional the biological reaction terms are quite simple since only production and mortality of phytoplanckton are represented. The model we propose here seems to be more realistic and has already been numerically validated using observations from the DYFAMED time-series station (Lévy et al. 17, Faugeras et al. ${ }^{12}$ ).

If data are regular enough the semigroup method can enable the existence of classical solutions to be proved. However it does not enable parabolic equations with time-dependent irregular coefficients to be easily handled. Since this is the case in the NPZD-model we consider a variational formulation approach is more attractive. The main purpose of this paper is to address the issue of the existence of weak solutions to this particular one-dimensional model. The method we propose is inspired from the work of Artola ${ }^{2}$ in which an existence result for a semilinear parabolic system is derived using a fixed-point argument. We introduce an approximate model and prove the existence of weak solutions to this model using this method. We then pass to the limit in the approximate model to prove the existence of weak solutions to the NPZD-model. Furthermore, as the variables of the model represent concentrations they should be positive. We show this is the case. 
We shall now briefly outline the contents of the paper. In the next section we introduce the equations of the one-dimensional NPZD-model and give some comments on the different parameterizations used. In Section 3 we set the mathematical framework and state our main result, which is proved in Sections 4 and 5 . The goal of Section 6 is twofold. First we show that the existence and positivity results still hold when different parameterizations found in the literature are used. Second, we address the issue of uniqueness of solutions. In order to prove uniqueness we need the nonlinear reaction terms to satisfy a local Lipschitz condition. We show this is the case in our particular model.

\section{Presentation of the one-dimensional NPZD-model}

\subsection{Equations of the model}

In this section we give the equations of the one-dimensional NPZD-model and formulate the initial-boundary value problem which will be studied.

Let us first of all justify the use of a one-dimensional model. We have in mind numerical studies (Faugeras et al. ${ }^{12}$, Lévy et al. ${ }^{16}$, Mémery et al. ${ }^{22}$ ) conducted with such one-dimensional models. In these studies simulations are forced with physical data (wind stress, heat fluxes, evaporation-precipitation) and validated by comparison with biogeochemical data (chlorophyll and nitrate) collected at the DYFAMED station. This station is located in the Northwestern Mediterranean Sea and is an interesting test case for several reasons. First, several biogeochemical production regimes that take place in the world ocean are found here. Secondly, the station is far enough away from the Ligurian Current to be sufficiently protected from lateral transport, thereby permitting a one-dimensional study.

In the above cited numerical studies the biogeochemical model is integrated in a one-dimensional physical model, which simulates the time evolution of velocity, temperature, salinity and turbulent kinetic energy (TKE). Advection is neglected even though this might result in a crude approximation in summer during strong wind events (Andersen and Prieur ${ }^{1}$ ). The only dynamic process which is taken into account is vertical diffusion.

The one-dimensional NPZD-model consists of four coupled semilinear parabolic equations. Before introducing them let us give some notations. In all the following we denote the nutrient, phytoplankton, zooplankton and detritus concentration vector by,

$$
\mathbf{C}=(N, P, Z, D)=\left(C_{1}, C_{2}, C_{3}, C_{4}\right),
$$

and the reaction terms by,

$$
\mathbf{f}=\left(f_{N}, f_{P}, f_{Z}, f_{D}\right)=\left(f_{1}, f_{2}, f_{3}, f_{4}\right) .
$$

The equations of the NPZD-model read as follows. For $i=1$ to 4 : 


$$
\begin{cases}\frac{\partial C_{i}}{\partial t}-\frac{\partial}{\partial x}\left(d(t, x) \frac{\partial C_{i}}{\partial x}\right)+\delta_{i, 4} v_{d} \frac{\partial C_{i}}{\partial x}=f_{i}(t, x, \mathbf{C}), & t \in] 0, T], \quad x \in] 0, L[, \\ \frac{\partial C_{i}}{\partial x}(t, 0)=\frac{\partial C_{i}}{\partial x}(t, L)=0, & t \in] 0, T], \\ C_{i}(0, x)=C_{i}^{0}(x), & x \in] 0, L[,\end{cases}
$$

with

$$
\left\{\begin{aligned}
f_{N}(t, x, \mathbf{C})= & \left(-\mu_{p}(1-\gamma) L_{I}(t, x, P) L_{N} P+\mu_{z} Z+\mu_{d} D\right) \mathbb{1}_{] 0, l]}(x) \\
& +(\tau(P+Z+D)) \mathbb{1}_{l l, L[}(x), \\
f_{P}(t, x, \mathbf{C})= & \left(\mu_{p}(1-\gamma) L_{I}(t, x, P) L_{N} P-G_{P} Z-m_{p} P\right) \mathbb{1}_{] 0, l]}(x) \\
& +(-\tau P) \mathbb{1}_{] l, L[}(x), \\
f_{Z}(t, x, \mathbf{C})= & \left(a_{p} G_{P} Z+a_{d} G_{D} Z-m_{z} Z-\mu_{z} Z\right) \mathbb{1}_{] 0, l]}(x) \\
& +(-\tau Z) \mathbb{1}_{l, L[}(x), \\
f_{D}(t, x, \mathbf{C})= & \left(\left(1-a_{p}\right) G_{P} Z-a_{d} G_{D} Z+m_{p} P+m_{z} Z-\mu_{d} D\right) \mathbb{1}_{] 0, l]}(x) \\
& +(-\tau D) \mathbb{1}_{l l, L}(x) .
\end{aligned}\right.
$$

$T$ is a fixed time. In numerical simulations, system (2.1) is intregated over a period of time which can vary from one month to a few years.

$L$ is the depth of the water column under consideration $(L \approx 1000 \mathrm{~m}), l$ is the maximum depth of the euphotic layer $(l \approx 200 \mathrm{~m})$.

$\mathbb{1}_{10, l]}$ and $\mathbb{1}_{] l, L[}$ are the usual indicator functions,

$$
\mathbb{1}_{] 0, l]}(x)= \begin{cases}1 & \text { if } x \in] 0, l], \\ 0 & \text { otherwise }\end{cases}
$$

$\delta_{i, 4}$ is the Kronecker symbol,

$$
\delta_{i, 4}= \begin{cases}1 & \text { if } \quad i=4 \\ 0 & \text { otherwise }\end{cases}
$$

Neuman boundary conditions at $x=0$ and $x=L$ express the fact that there is no flux through the surface of the ocean and through the ocean floor.

Initial concentrations, $C_{i}^{0}$, satisfy $C_{i}^{0}(x) \geq 0$ for all $\left.x \in\right] 0, L[$.

The different parameters which appear in the reaction terms $f_{i}$ are strictly positive constants. All of them are shown in Table 1. A schematic representation of 
the model is shown on Figure 1 . Let us note that parameters $\gamma, a_{p}$ and $a_{d}$ satisfy $1-\gamma>0,1-a_{p}>0$ and $1-a_{d}>0$.

The nonlinear functions $L_{I}, L_{N}, G_{P}$ and $G_{D}$ are given explicitly in the following subsection, and more details about the model can be found in Lévy et al. ${ }^{17}$.

Table 1: Parameter values

\begin{tabular}{|l|c|c|c|}
\hline parameter & name & value & unit \\
\hline half-saturation constant & $k_{n}$ & 0.5 & molN $^{-3}$ \\
maximal grazing rate & $g_{z}$ & 0.75 & day $^{-1}$ \\
half-saturation constant for grazing & $k_{z}$ & 1 & molN.m $^{-3}$ \\
assimilated fraction of phytoplankton & $a_{p}$ & 0.7 & \\
assimilated fraction of detritus & $a_{d}$ & 0.5 & \\
zooplankton excretion rate & $\mu_{z}$ & 0.1 & day $^{-1}$ \\
phytoplankton mortality rate & $m_{p}$ & 0.03 & day $^{-1}$ \\
zooplankton mortality rate & $m_{z}$ & 0.03 & day $^{-1}$ \\
detritus remineralization rate & $\mu_{d}$ & 0.09 & day $^{-1}$ \\
detritus sedimentation speed & $v_{d}$ & 5 & m.day $^{-1}$ \\
maximal growth rate & $\mu_{p}$ & 2 & day $^{-1}$ \\
exsudation fraction & $\gamma$ & 0.05 & \\
remineralization rate & $\tau$ & 0.05 & day $^{-1}$ \\
\hline
\end{tabular}

Figure 1: Schematic representation of the compartments and processes of the NPZD surface layer model.

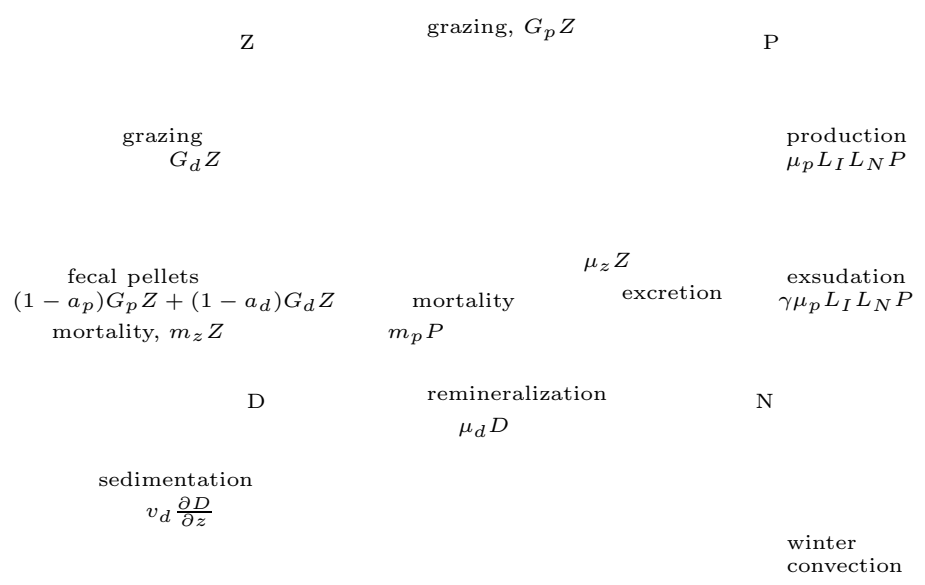


Well-posedness of a coupled biological-physical model for the upper ocean

\subsection{Comments and hypotheses}

1. The mixing or diffusion coefficient, $d(t, x)$, is obtained diagnostically from TKE (Gaspar et al. ${ }^{14}$ ). In modeling studies it is considered in the first approximation that biological variables do not influence physical variables. As a consequence biological tracers are vertically mixed with the same coefficient as temperature and salinity. This coefficient is an output of the physical model and data for the biological model. Consequently it does not depend on $\mathbf{C}$. We are thus given once and for all a mixing coefficient $d(t, x)$. It strongly varies in space and time and we can not assume it is particularly regular (Lewandosky $\left.{ }^{18}\right)$. The usual basic assumption made in the mathematical literature as well as in numerical studies is the following. We suppose that

$$
\left.0<d_{0} \leq d(t, x) \leq d_{\infty}, \text { a.e. in }\right] 0, T[\times] 0, L[.
$$

2. Because of functions $\mathbb{1}_{00, l]}$ and $\mathbb{1}_{l l, L[}$, the equations of the model are not the same above and below the depth $l$ which physically corresponds to the depth at which the action of light on the system becomes negligeable. This corresponds to a discontinuity of the reaction terms $f_{i}(t, x, \mathbf{C})$ at the point $x=l$. It is the choice of modelization made by Lévy et al. ${ }^{17}$. Above the depth $l$ the reaction terms correspond to the schematic representation of the model shown on Figure 1. The basic biogeochemical fluxes are represented using a minimum number of prognostic variables. Nutrients allow the estimation of production to be made. Zooplankton mortality and detrital sedimentation feed the particle export flux. Below the depth $l$ remineralization processes are preponderent and the surface model does not apply. Instead decay of phytoplankton, zooplankton and detritus in nutrients parameterize remineralization. More details about the modeled biogeochemical processes can be found in Lévy et al. ${ }^{17}$. In the following points we give the analytical expression of the nonlinear terms which are used.

3. $L_{N}, G_{P}$ and $G_{D}$ are nonlinear functions.

- $L_{N}$ parameterizes the nutrient limitation on phytoplankton growth. It follows the Michaelis-Menten kinetic, $L_{N}=\frac{N}{k_{n}+N}$. The possible nullification of the term $k_{n}+N$, invites us to define, $L_{N}=\frac{N}{k_{n}+|N|}$. This formulation will be used in the following. We will show that if initial concentrations are positive then concentrations always stay positive, thus the two formulations are equivalent. Let us remark that,

$$
\begin{aligned}
& L_{N} \text { is defined and continuous on } \mathbb{R}, \\
& \left|L_{N}(N)\right| \leq 1, \forall N \in \mathbb{R} .
\end{aligned}
$$


- $G_{P}$ and $G_{D}$ are the zooplankton grazing rates on phytoplankton and detritus. The formulation used is a squared Michaelis-Menten response function:

$$
\begin{aligned}
G_{P} & =\frac{g_{z} P^{2}}{k_{z}+P^{2}}, \\
G_{D} & =\frac{g_{z} D^{2}}{k_{z}+D^{2}} .
\end{aligned}
$$

In the remainder of this paper we use the following properties:

$G_{P}$ and $G_{D}$ are defined and continuous on $\mathbb{R}$,

$\left|G_{P}\right| \leq g_{z}, \forall P \in \mathbb{R}$,

$\left|G_{D}\right| \leq g_{z}, \forall D \in \mathbb{R}$.

4. The limitation of phytoplankton growth by light is parameterized by,

$$
L_{I}(t, x, P)=1-\exp \left(-P A R(t, x, P) / k_{p a r}\right),
$$

$k_{p a r}$ is a positive constant. The photosynthetic available radiation, $P A R$, is predicted from surface irradiance and phytoplankton pigment content by a light absorption model according to Lévy et al. ${ }^{17}$. From a biological point of view, the fact that $P A R$ depends on $P$ is important. This models the so-called self-shading effect. We give further details of the parameterization of $P A R$ in Section 6, and here we only suppose it is a positive function, continuous in $P$ for a.e. $t, x$ and measurable in $t, x$ for all $P$. In order to prove the existence result we have to notice that:

$$
\begin{aligned}
& L_{I} \text { is defined on }[0, T] \times[0, L] \times \mathbb{R}, \\
& 0 \leq L_{I}(t, x, P) \leq 1, \text { a.e in }[0, T] \times[0, L] \times \mathbb{R}, \\
& (t, x) \rightarrow L_{I}(t, x, P) \text { is measurable, for all } P \in \mathbb{R}, \\
& P \rightarrow L_{I}(t, x, P) \text { is continuous, for a.e }(t, x) \in[0, T] \times[0, L] .
\end{aligned}
$$

5. Eventually, let us remark the presence of the advection term $v_{d} \frac{\partial D}{\partial x}$ in the detritus equations. Detritus, $D$, sink at a speed of $v_{d}$.

\section{Mathematical preliminaries and statement of main result}

\subsection{Functional spaces}

In this section we introduce the functional spaces which we use in the remainder of this work. All this study is conducted on the open set $] 0, L[$ and $T$ is a fixed time. Throughout this work, concentrations, $C_{i}$, are considered as elements of the functional space $L^{2}(0, L)$ whose Hilbert space structure is convenient to use. However, let us remember that $L^{2}(0, L)$ is continuously imbedded into $L^{1}(0, L)$ which is a natural space for concentrations. 
$\mathbf{H}$ and $\mathbf{H}^{1}$ are the separable Hilbert spaces defined by

$$
\begin{aligned}
& \mathbf{H}=\left(L^{2}(0, L)\right)^{4}, \\
& \mathbf{H}^{1}=\left(H^{1}(0, L)\right)^{4} .
\end{aligned}
$$

$\mathbf{H}$ is equipped with the scalar product

$$
\begin{aligned}
(\mathbf{C}, \hat{\mathbf{C}}) & =\int_{0}^{L} \sum_{i=1}^{4} C_{i}(x) \hat{C}_{i}(x) d x \\
& =\sum_{i=1}^{4}\left(C_{i}, \hat{C}_{i}\right)_{L^{2}(0, L)} .
\end{aligned}
$$

We denote by $\|$.$\| the induced norm on \mathbf{H}$.

$\mathbf{H}^{1}$ is equipped with the scalar product

$$
\begin{aligned}
(\mathbf{C}, \hat{\mathbf{C}})_{1} & =\int_{0}^{L} \sum_{i=1}^{4} C_{i}(x) \hat{C}_{i}(x) d x+\int_{0}^{L} \sum_{i=1}^{4} \frac{\partial C_{i}(x)}{\partial x} \frac{\partial \hat{C}_{i}(x)}{\partial x} d x \\
& =\sum_{i=1}^{4}\left(C_{i}, \hat{C}_{i}\right)_{L^{2}(0, L)}+\sum_{i=1}^{4}\left(\frac{\partial C_{i}}{\partial x}, \frac{\partial \hat{C}_{i}}{\partial x}\right)_{L^{2}(0, L)} .
\end{aligned}
$$

We denote by $\|.\|_{1}$, the induced norm on $\mathbf{H}^{1}$.

We will also have to consider the space $\mathbf{L}^{\infty}=\left(L^{\infty}(0, L)\right)^{4} . L^{\infty}(0, L)$ is a Banach equipped with the norm

$$
\left\|C_{i}\right\|_{\infty}=\inf \left\{M ;\left|C_{i}(x)\right| \leq M \text { a.e. in }(0, L)\right\} .
$$

Similarly $\mathbf{L}^{\infty}$ is a Banach space equipped with the norm

$$
\|\mathbf{C}\|_{\infty}=\sup _{i=1, \ldots, 4}\left\|C_{i}\right\|_{\infty} .
$$

Now, if $X$ is a real Banach space equipped with the norm $\|.\|_{X}, C([0, T], X)$ is the space of continuous functions on $[0, T]$ with values in $X$, equipped with the norm,

$$
\|\mathbf{C}\|_{C([0, T], X)}=\sup _{[0, T]}\|\mathbf{C}(t)\|_{X}
$$

Similarly $L^{2}(0, T, X)$ is the space of functions $L^{2}$ in time with values in $X$, equipped with the norm,

$$
\|\left.\mathbf{C}\right|_{L^{2}(0, T, X)}=\left(\int_{0}^{T}\|\mathbf{C}(t)\|_{X}^{2} d t\right)^{1 / 2},
$$

and $L^{\infty}(0, T, X)$ is the space of functions $L^{\infty}$ in time with values in $X$, equipped with the norm,

$$
\|\mathbf{C}\|_{L^{\infty}(0, T, X)}=\inf \left\{M ;\|\mathbf{C}(t)\|_{X} \leq M \text { a.e in }(0, T)\right\} .
$$

$C([0, T], X), L^{2}(0, T, X)$ and $L^{\infty}(0, T, X)$ are Banach spaces.

We have the useful 
Lemma 3.1 The imbedding, $\mathbf{H}^{1} \subset \mathbf{L}^{\infty}$, is continuous.

The imbeddings, $\mathbf{H}^{1} \subset \mathbf{H}$ and $\mathbf{H}^{1} \subset C\left([0, L], \mathbb{R}^{4}\right)$ are compact.

Proof. It is a consequence of corollaries IX.14 and IX.16 in Brézis ${ }^{5}$, and of the Rellich-Kondrachoff theorem (Lions and Magenes ${ }^{20}$ ) $\square$.

$\mathbf{H}^{\prime}$ denotes the dual of $\mathbf{H}$ and $\left(\mathbf{H}^{1}\right)^{\prime}$ the dual of $\mathbf{H}^{1}$. When $\mathbf{H}$ is identified with its dual, we have the classical scheme,

$$
\mathbf{H}^{1} \subset \mathbf{H}=\mathbf{H}^{\prime} \subset\left(\mathbf{H}^{1}\right)^{\prime},
$$

where each space is dense in the following and the imbeddings are continuous.

Let us denote by $W\left(\mathbf{H}^{1}\right)$ the Hilbert space,

$$
W\left(\mathbf{H}^{1}\right)=\left\{\mathbf{C} \in L^{2}\left(0, T, \mathbf{H}^{1}\right) ; \frac{d \mathbf{C}}{d t} \in L^{2}\left(0, T,\left(\mathbf{H}^{1}\right)^{\prime}\right)\right\} .
$$

Lemma 3.2 Every $\mathbf{C} \in W\left(\mathbf{H}^{1}\right)$ is a.e equal to a continuous function from $[0, T]$ to $\mathbf{H}$. Moreover we have the following continuous imbedding,

$$
W\left(\mathbf{H}^{1}\right) \subset C([0, T], \mathbf{H}) .
$$

Proof. See Dautray and Lions ${ }^{7}$ for example $\square$.

Moreover, because the injective mapping $\mathbf{H}^{1} \subset \mathbf{H}$ is compact, we know that,

Lemma 3.3 The identity mapping, $W\left(\mathbf{H}^{1}\right) \subset L^{2}(0, T, \mathbf{H})$, is compact.

Proof. See Aubin ${ }^{3}$ or Lions ${ }^{19} \square$.

\subsection{A preliminary transformation of the system and the bilinear form} $a\left(t, \mathbf{C}, \mathbf{C}^{\prime}\right)$

In order to work with a bilinear form as simple as possible, we start by adding $\lambda C_{i}$ to both sides of system (2.1). The value of $\lambda>0$ will be fixed in what follows. This leads to the equivalent system, for $i=1$ to 4 :

$$
\begin{cases}\frac{\partial C_{i}}{\partial t}-\frac{\partial}{\partial x}\left(d(t, x) \frac{\partial C_{i}}{\partial x}\right)+\delta_{i, 4} v_{d} \frac{\partial C_{i}}{\partial x}+\lambda C_{i} & \\ =f_{i}(t, x, \mathbf{C})+\lambda C_{i}, & t \in] 0, T], x \in] 0, L[, \\ \frac{\partial C_{i}}{\partial x}(t, 0)=\frac{\partial C_{i}}{\partial x}(t, L)=0, & t \in] 0, T], \\ C_{i}(0, x)=C^{0}(x), & x \in] 0, L[\end{cases}
$$


For $N, N^{\prime}, P, P^{\prime}, Z, Z^{\prime}$ and $D, D^{\prime} \in H^{1}(0, L)$, we define

$$
\begin{aligned}
& a_{N}\left(t, N, N^{\prime}\right)=\int_{0}^{L} d(t, x) \frac{\partial N}{\partial x} \frac{\partial N^{\prime}}{\partial x}+\lambda \int_{0}^{L} N N^{\prime}, \\
& a_{P}\left(t, P, P^{\prime}\right)=\int_{0}^{L} d(t, x) \frac{\partial P}{\partial x} \frac{\partial P^{\prime}}{\partial x}+\lambda \int_{0}^{L} P P^{\prime}, \\
& a_{Z}\left(t, Z, Z^{\prime}\right)=\int_{0}^{L} d(t, x) \frac{\partial Z}{\partial x} \frac{\partial Z^{\prime}}{\partial x}+\lambda \int_{0}^{L} Z Z^{\prime}, \\
& a_{D}\left(t, D, D^{\prime}\right)=\int_{0}^{L} d(t, x) \frac{\partial D}{\partial x} \frac{\partial D^{\prime}}{\partial x}+\int_{0}^{L} v_{d} \frac{\partial D}{\partial x} D^{\prime}+\lambda \int_{0}^{L} D D^{\prime},
\end{aligned}
$$

and

$$
a\left(t, \mathbf{C}, \mathbf{C}^{\prime}\right)=a_{N}\left(t, N, N^{\prime}\right)+a_{P}\left(t, P, P^{\prime}\right)+a_{Z}\left(t, Z, Z^{\prime}\right)+a_{D}\left(t, D, D^{\prime}\right) .
$$

Lemma 3.4 For a.e. $t \in[0, T], a\left(t, \mathbf{C}, \mathbf{C}^{\prime}\right)$ is a continuous bilinear form on $\mathbf{H}^{1} \times$ $\mathbf{H}^{1}$. For all $\mathbf{C}, \mathbf{C}^{\prime} \in \mathbf{H}^{1}, t \rightarrow a\left(t, \mathbf{C}, \mathbf{C}^{\prime}\right)$ is measurable and there exists a constant $M_{a}>0$ such that,

$$
\left|a\left(t, \mathbf{C}, \mathbf{C}^{\prime}\right)\right| \leq M_{a}\|\mathbf{C}\|_{1}\left\|\mathbf{C}^{\prime}\right\|_{1}, \quad \forall \mathbf{C}, \mathbf{C}^{\prime} \in \mathbf{H}^{1} .
$$

For a fixed $\lambda, \lambda \geq \frac{v_{d}^{2}}{2 d_{0}}$, there exists a constant $c_{0}>0$ such that,

$$
a(t, \mathbf{C}, \mathbf{C}) \geq c_{0}\|\mathbf{C}\|_{1}^{2}, \quad \forall t \in[0, T], \quad \forall \mathbf{C} \in \mathbf{H}^{1} .
$$

Proof. The proof for this is classical and it is omited $\square$.

\subsection{The reaction terms and the nonlinear operator $\mathrm{G}$}

In this paragraph we show that the reaction terms of the NPZD-model enable us to define a continuous operator $\mathbf{G}$ on $L^{2}(0, T, \mathbf{H})$.

Lemma 3.5 The reaction terms $f_{N}, f_{P}, f_{Z}$ and $f_{D}$ defined in Section 2 have the following properties:

(P1) For a.e. $(t, x) \in[0, T] \times[0, L]$, and all $\mathbf{C} \in \mathbb{R}^{4}$,

$$
\begin{aligned}
& \left|f_{N}(t, x, \mathbf{C})\right| \leq\left(\mu_{p}(1-\gamma)+\tau\right)|P|+\left(\mu_{z}+\tau\right)|Z|+\left(\mu_{d}+\tau\right)|D|, \\
& \left|f_{P}(t, x, \mathbf{C})\right| \leq\left(\mu_{p}(1-\gamma)+m_{p}+\tau\right)|P|+g_{z}|Z|, \\
& \left|f_{Z}(t, x, \mathbf{C})\right| \leq\left(\left(a_{p}+a_{d}\right) g_{z}+m_{z}+\mu_{z}+\tau\right)|Z|, \\
& \left|f_{D}(t, x, \mathbf{C})\right| \leq\left(\left(\left(1-a_{p}\right)+a_{d}\right) g_{z}+m_{z}\right)|Z|+m_{p}|P|+\left(\mu_{d}+\tau\right)|D| .
\end{aligned}
$$

(P2) The function $\mathbf{f}(t, x, \mathbf{C})$, defined from $[0, T] \times[0, L] \times \mathbb{R}^{4} \rightarrow \mathbb{R}^{4}$, is measurable in $(t, x)$, for all $\mathbf{C} \in \mathbb{R}^{4}$, and is continuous in $\mathbf{C}$, for a.e. $(t, x) \in[0, T] \times[0, L]$. 
Proof. The proof is straightforward and uses the comments of Section $2.2 \square$.

We now define a function $\mathbf{g}(t, x, \mathbf{C})=\mathbf{f}(t, x, \mathbf{C})+\lambda \mathbf{C}$ from $[0, T] \times[0, L] \times \mathbb{R}^{4} \rightarrow$ $\mathbb{R}^{4}$ and a nonlinear operator, $\mathbf{G}$, by:

$$
\mathbf{G C}=\mathbf{g}(t, x, \mathbf{C}(t, x)), \quad(t, x) \in[0, T] \times[0, L] .
$$

Proposition 3.1 The operator, $\mathbf{G}$, is well defined from $L^{2}(0, T, \mathbf{H})$ to itself. There exists a constant $M_{g}>0$, depending only on the parameters of the model, such that, for all $\mathbf{C} \in E^{2}(0, T, \mathbf{H})$

$$
\|\mathbf{G C}\|_{L^{2}(0, T, \mathbf{H})} \leq M_{g}\|\mathbf{C}\|_{L^{2}(0, T, \mathbf{H})} .
$$

The operator $\mathbf{G}$ is continuous on $L^{2}(0, T, \mathbf{H})$.

Proof. Let $\mathbf{C} \in L^{2}(0, T, \mathbf{H})$ and $t \in[0, T]$. From point $(P 1)$ of lemma 3.5 we obtain,

$$
\begin{aligned}
& \|\mathbf{G C}(t)\|^{2}= \\
& \int_{0}^{L}\left|f_{N}(t, x, \mathbf{C}(t, x))+\lambda N(t, x)\right|^{2}+\left|f_{P}(t, x, \mathbf{C}(t, x))+\lambda P(t, x)\right|^{2} \\
& +\left|f_{Z}(t, x, \mathbf{C}(t, x))+\lambda Z(t, x)\right|^{2}+\left|f_{D}(t, x, \mathbf{C}(t, x))+\lambda D(t, x)\right|^{2} d x, \\
& \|\mathbf{G C}(t)\|^{2} \leq \\
& \operatorname{cte}_{1}\left(\|P(t)\|_{L^{2}(0, L)}^{2}+\|Z(t)\|_{L^{2}(0, L)}^{2}+\|D(t)\|_{L^{2}(0, L)}^{2}+\|N(t)\|_{L^{2}(0, L)}^{2}\right) \\
& +c t e_{2}\left(\|P(t)\|_{L^{2}(0, L)}^{2}+\|Z(t)\|_{L^{2}(0, L)}^{2}+\|P(t)\|_{L^{2}(0, L)}^{2}\right) \\
& +c t e_{3}\left(\|Z(t)\|_{L^{2}(0, L)}^{2}\right) \\
& +c t e_{4}\left(\|Z(t)\|_{L^{2}(0, L)}^{2}+\|P(t)\|_{L^{2}(0, L)}^{2}+\|D(t)\|_{L^{2}(0, L)}^{2}\right), \\
& \|\mathbf{G C}(t)\|^{2} \leq M_{g}^{2}\|\mathbf{C}(t)\|^{2},
\end{aligned}
$$

and integrating on $[0, T]$,

$$
\|\mathbf{G C}\|_{L^{2}(0, T, \mathbf{H})} \leq M_{g}\|\mathbf{C}\|_{L^{2}(0, T, \mathbf{H})} .
$$

From point $(P 2)$ of lemma 3.5 we know that the function,

$$
\mathbf{g}(t, x, \mathbf{C})=\mathbf{f}(t, x, \mathbf{C})+\lambda \mathbf{C},
$$

from $[0, T] \times[0, L] \times \mathbb{R}^{4} \rightarrow \mathbb{R}^{4}$, satisfies the conditions of Carathéodory and by theorem 2.1 page 22 of Krasnosel'skii ${ }^{15}$, we know that the operator $\mathbf{G}$ is continuous $\square$.

\subsection{Variational formulation}

We can now write the definition of a weak solution to system (2.1), 
Definition 3.1 $\mathbf{C} \in W\left(\mathbf{H}^{1}\right)$ is a weak solution of system (2.1) if

$$
\forall \phi \in \mathbf{H}^{1}, \quad\left(\frac{d \mathbf{C}}{d t}, \phi\right)+a(t, \mathbf{C}, \phi)=(\mathbf{G C}, \phi),
$$

in the $\mathcal{D}^{\prime}(] 0, T[)$ sens, and $\mathbf{C}(0)=\mathbf{C}^{0}$.

and state the main result of this paper,

Theorem 3.1 Let $\mathbf{C}^{0} \in \mathbf{H}$. There exists a weak solution to system (2.1). Furthermore, if $N^{0}, P^{0}, Z^{0}$ and $D^{0}$ are positive then $N, P, Z$ and $D$ are positive for a.e. $t \in[0, T]$.

The proof is given in the next two sections.

\section{Existence}

The existence result is obtained in two steps. We first define an approximate problem, in which the operator $\mathbf{G}$ is approximated by an operator $\mathbf{G}_{n}$. This approximate problem is solved using the Schauder fixed-point theorem. In the second step we let $n \rightarrow \infty$ to obtain a solution to the initial problem.

\subsection{Step 1: approximated problem}

Let $n>0$ be a fixed integer and $\mathbf{g}_{n}$ be defined by,

$$
\begin{aligned}
\mathbf{g}_{n}: \quad[0, T] \times[0, L] \times \mathbb{R}^{4} & \rightarrow \mathbb{R}^{4} \\
(t, x, \mathbf{C}) & \rightarrow\left(\frac{(\mathbf{g}(t, x, \mathbf{C}))_{i}}{1+\frac{1}{n}\left|(\mathbf{g}(t, x, \mathbf{C}))_{i}\right|}\right)_{i=1, \ldots 4 .}
\end{aligned}
$$

Define the nonlinear operator, $\mathbf{G}_{n}$, by:

$$
\mathbf{G}_{n} \mathbf{C}=\mathbf{g}_{n}(t, x, \mathbf{C}(t, x)), \quad(t, x) \in[0, T] \times[0, L] .
$$

Proposition 4.2 The operator, $\mathbf{G}_{n}$, is well defined from $L^{2}(0, T, \mathbf{H})$ to itself and there exists a constant, $M_{g}>0$, such that for all $\mathbf{C} \in E^{2}(0, T, \mathbf{H})$,

$$
\left\|\left.\mathbf{G}_{n} \mathbf{C}\right|_{L^{2}(0, T, \mathbf{H})} \leq M_{g}\right\| \mathbf{C} \|_{L^{2}(0, T, \mathbf{H})} .
$$

The operator $\mathbf{G}_{n}$ is continuous on $L^{2}(0, T, \mathbf{H})$.

For all $\mathbf{C} \in L^{2}(0, T, \mathbf{H})$, we also have the estimation,

$$
\left\|\mathbf{G}_{n} \mathbf{C}\right\|_{L^{2}(0, T, \mathbf{H})} \leq 2 n \sqrt{L T} .
$$

Proof. Let $\mathbf{C} \in L^{2}(0, T, \mathbf{H})$. From the definition of $\mathbf{g}_{n}$ and from proposition 3.1 we obtain,

$$
\left\|\mathbf{G}_{n} \mathbf{C}\right\|_{L^{2}(0, T, \mathbf{H})} \leq\|\mathbf{G C}\|_{L^{2}(0, T, \mathbf{H})} \leq M_{g}\|\mathbf{C}\|_{L^{2}(0, T, \mathbf{H})} .
$$

The estimation $\|\left.\mathbf{G}_{n} \mathbf{C}\right|_{L^{2}(0, T, \mathbf{H})} \leq 2 n \sqrt{L T}$ is also derived easily from the choice made to define $\mathbf{g}_{n}$. 
As in proposition 3.1, $\mathbf{g}_{n}$ satisfies the conditions of Carathéodory and $\mathbf{G}_{n}$ is continuous on $L^{2}(0, T, \mathbf{H})$

We now seek a solution to the approximated system and show that such a solution is a fixed-point of the operator $\Theta$ defined in the next proposition.

Proposition 4.3 Let $\hat{\mathbf{C}}$ be a fixed element of $L^{2}(0, T, \mathbf{H})$ and let $\mathbf{C}^{0} \in \mathbf{H}$. There exists a unique solution to the problem:

find $\mathbf{C} \in W\left(\mathbf{H}^{1}\right)$ such that,

$$
\forall \phi \in \mathbf{H}^{1}, \quad\left(\frac{d \mathbf{C}}{d t}, \phi\right)+a(t, \mathbf{C}, \phi)=\left(\mathbf{G}_{n} \hat{\mathbf{C}}, \phi\right),
$$

in the $\mathcal{D}^{\prime}(] 0, T[)$ sens,

and $\mathbf{C}(0)=\mathbf{C}^{0}$.

This solution defines an operator $\Theta$ on $L^{2}(0, T, \mathbf{H}), \Theta \hat{\mathbf{C}}=\mathbf{C}$.

Proof. Since the problem is linear in $\mathbf{C}$ and $\mathbf{G}_{n} \hat{\mathbf{C}}$ is fixed in $L^{2}(0, T, \mathbf{H})$, the proof is classical (e.g. Dautray and Lions, $\left.{ }^{7}\right) \square$.

To insure that $\Theta$ has a fixed point, we show that the Schauder fixed point theorem can be applied.

Lemma 4.6 The operator $\Theta$ is continuous on $L^{2}(0, T, \mathbf{H})$.

Proof. Let $\hat{\mathbf{C}}^{1}$ and $\hat{\mathbf{C}}^{2} \in L^{2}(0, T, \mathbf{H}) . \mathbf{C}^{1}$ and $\mathbf{C}^{2}$, the associated solutions to the problem of proposition 4.3 , satisfy,

$$
\left(\frac{d}{d t}\left(\mathbf{C}^{1}-\mathbf{C}^{2}\right), \phi\right)+a\left(t, \mathbf{C}^{1}-\mathbf{C}^{2}, \phi\right)=\left(\mathbf{G}_{n} \hat{\mathbf{C}}^{1}-\mathbf{G}_{n} \hat{\mathbf{C}}^{2}, \phi\right) .
$$

Taking $\phi=\mathbf{C}^{1}-\mathbf{C}^{2}$ as a test function, integrating on $[0, t]$, using the coerciveness of $a$ and Cauchy-Schwarz inequality, we obtain,

$$
\begin{aligned}
& \int_{0}^{t} \frac{1}{2} \frac{d}{d t}\left\|\mathbf{C}^{1}(s)-\mathbf{C}^{2}(s)\right\|^{2}+c_{0}\left\|\mathbf{C}^{1}(s)-\mathbf{C}^{2}(s)\right\|_{1}^{2} d s \\
& \leq \int_{0}^{t}\left\|\mathbf{G}_{n} \hat{\mathbf{C}}^{1}(s)-\mathbf{G}_{n} \hat{\mathbf{C}}^{2}(s)\right\|\left\|\mathbf{C}^{1}(s)-\mathbf{C}^{2}(s)\right\| d s .
\end{aligned}
$$

As $\mathbf{C}^{1}(0)=\mathbf{C}^{2}(0)=\mathbf{C}^{0}$ we obtain using Young's inequality,

$$
\begin{aligned}
& \left\|\mathbf{C}^{1}(t)-\mathbf{C}^{2}(t)\right\|^{2}+\int_{0}^{t} 2 c_{0}\left\|\mathbf{C}^{1}(s)-\mathbf{C}^{2}(s)\right\|_{1}^{2} d s \\
& \leq \int_{0}^{t} \frac{1}{\alpha}\left\|\mathbf{G}_{n} \hat{\mathbf{C}}^{1}(s)-\mathbf{G}_{n} \hat{\mathbf{C}}^{2}(s)\right\|^{2} d s+\alpha \int_{0}^{t}\left\|\mathbf{C}^{1}(s)-\mathbf{C}^{2}(s)\right\|^{2} d s,
\end{aligned}
$$

and with $\alpha=2 c_{0}$,

$$
\left\|\mathbf{C}^{1}(t)-\mathbf{C}^{2}(t)\right\|^{2} \leq \int_{0}^{T} \frac{1}{2 c_{0}}\left\|\mathbf{G}_{n} \hat{\mathbf{C}}^{1}(s)-\mathbf{G}_{n} \hat{\mathbf{C}}^{2}(s)\right\|^{2} d s .
$$


Eventually, we obtain, integrating on $[0, T]$,

$$
\left\|\Theta \hat{\mathbf{C}}^{1}-\Theta \hat{\mathbf{C}}^{2}\right\|_{L^{2}(0, T, \mathbf{H})}=\left\|\mathbf{C}^{1}-\mathbf{C}^{2}\right\|_{L^{2}(0, T, \mathbf{H})} \leq \sqrt{\frac{T}{2 c_{0}}}\left\|\mathbf{G}_{n} \hat{\mathbf{C}}^{1}-\mathbf{G}_{n} \hat{\mathbf{C}}^{2}\right\|_{L^{2}(0, T, \mathbf{H})}
$$

and $\Theta$ is continuous as $\mathbf{G}_{n}$ is $\square$.

Lemma 4.7 The operator $\Theta$ maps $L^{2}(0, T, \mathbf{H})$ in the ball

$$
B=\left\{\mathbf{C} \in L^{2}(0, T, \mathbf{H}),\|\mathbf{C}\|_{L^{2}(0, T, \mathbf{H})} \leq \sqrt{T\left(\frac{2 L T n^{2}}{c_{0}}+\left\|\mathbf{C}^{0}\right\|^{2}\right)}\right\} .
$$

In particular, we have, $\Theta(B) \subset B$.

Proof. Let $\hat{\mathbf{C}} \in L^{2}(0, T, \mathbf{H})$. C , the solution to the problem of proposition 4.3, satisfies,

$$
\left(\frac{d}{d t} \mathbf{C}, \phi\right)+a(t, \mathbf{C}, \phi)=\left(\mathbf{G}_{n} \hat{\mathbf{C}}, \phi\right) .
$$

Taking $\phi=\mathbf{C}$ as a test function, integrating $[0, t]$, using the coerciveness of $a$ and the Cauchy-Schwarz inequality we obtain,

$$
\int_{0}^{t} \frac{1}{2} \frac{d}{d t}\|\mathbf{C}(s)\|^{2}+c_{0}\|\mathbf{C}(s)\|_{1}^{2} d s \leq \int_{0}^{t}\left\|\mathbf{G}_{n} \hat{\mathbf{C}}(s)\right\|\|\mathbf{C}(s)\| d s,
$$

with Young's inequality,

$$
\|\mathbf{C}(t)\|^{2}+\int_{0}^{t} 2 c_{0}\|\mathbf{C}(s)\|_{1}^{2} d s \leq \int_{0}^{t} \frac{1}{\alpha}\left\|\mathbf{G}_{n} \hat{\mathbf{C}}(s)\right\|^{2} d s+\alpha \int_{0}^{t}\|\mathbf{C}(s)\|^{2} d s+\left\|\mathbf{C}^{0}\right\|^{2},
$$

with $\alpha=2 c_{0}$ and as $\int_{0}^{t}\left\|\mathbf{G}_{n} \hat{\mathbf{C}}(s)\right\|^{2} d s \leq 4 L T n^{2}$, we have

$$
\|\mathbf{C}(t)\|^{2} \leq \frac{4 L T n^{2}}{2 c_{0}}+\left\|\mathbf{C}^{0}\right\|^{2}
$$

integrating once more on $[0, T]$ we obtain,

$$
\|\Theta \hat{\mathbf{C}}\|_{L^{2}(0, T, \mathbf{H})}^{2}=\|\mathbf{C}\|_{L^{2}(0, T, \mathbf{H})}^{2} \leq T\left(\frac{2 L T n^{2}}{c_{0}}+\left\|\mathbf{C}^{0}\right\|^{2}\right) .
$$

$\square$.

Lemma 4.8 The operator $\Theta$ is compact.

Proof. Let $B$ be a bounded set in $L^{2}(0, T, \mathbf{H})$. Let us show that $\Theta(B)$ is bounded in $W\left(\mathbf{H}^{1}\right)$. Let $\hat{\mathbf{C}} \in B \subset L^{2}(0, T, \mathbf{H})$ and let $\mathbf{C}$ be the associated solution to the problem of proposition 4.3. As in the proof of lemma 4.7 we obtain

$$
\|\mathbf{C}(t)\|^{2}+\int_{0}^{t} 2 c_{0}\|\mathbf{C}(s)\|_{1}^{2} d s \leq \int_{0}^{t} \frac{1}{\alpha}\left\|\mathbf{G}_{n} \hat{\mathbf{C}}(s)\right\|^{2} d s+\alpha \int_{0}^{t}\|\mathbf{C}(s)\|^{2} d s+\left\|\mathbf{C}^{0}\right\|^{2} .
$$


Taking $\alpha=c_{0}$ this time, we obtain

$$
\int_{0}^{T}\|\mathbf{C}(s)\|_{1}^{2} d s \leq \frac{1}{c_{0}}\left\|\mathbf{G}_{n} \hat{\mathbf{C}}\right\|_{L^{2}(0, T, \mathbf{H})^{2}}+\left\|\mathbf{C}^{0}\right\|^{2} \leq \frac{4 L T n^{2}}{c_{0}}+\left\|\mathbf{C}^{0}\right\|^{2},
$$

and $\Theta \hat{\mathbf{C}}$ is bounded in $L^{2}\left(0, T, \mathbf{H}^{1}\right)$.

Moreover we obtain

$$
\forall \phi \in \mathbf{H}^{1}, \quad\left(\frac{d \mathbf{C}}{d t}, \phi\right)+a(t, \mathbf{C}, \phi)=\left(\mathbf{G}_{n} \hat{\mathbf{C}}, \phi\right) .
$$

From lemma 3.4,

$$
\left|\left(\frac{d \mathbf{C}}{d t}, \phi\right)\right| \leq M_{a}\|\mathbf{C}\|_{1}\|\phi\|_{1}+\left\|\mathbf{G}_{n} \hat{\mathbf{C}} \mid\right\|\|\phi\| \leq M_{a}\|\mathbf{C}\|_{1}\|\phi\|_{1}+2 n \sqrt{L}\|\phi\|_{1},
$$

and

$$
\int_{0}^{T}\left\|\frac{d \mathbf{C}}{d t}\right\|_{\left(\mathbf{H}^{1}\right)^{\prime}}^{2} d s \leq 2 \int_{0}^{T}\left(4 L n^{2}+M^{2}\|\mathbf{C}\|_{1}^{2}\right) d s .
$$

And therefore, $\left\|\frac{d \mathbf{C}}{d t}\right\|_{L^{2}\left(0, T,\left(\mathbf{H}^{1}\right)^{\prime}\right)}$ is bounded in $L^{2}\left(0, T,\left(\mathbf{H}^{1}\right)^{\prime}\right)$.

The range of $\Theta$ is in $W\left(\mathbf{H}^{1}\right)$, from lemma 3.3, the injection $W\left(\mathbf{H}^{1}\right) \subset L^{2}(0, T, \mathbf{H})$ is compact, and this concludes the proof $\square$.

It is now possible to state the main result of this section, concerning the existence of weak solutions to the approximated problem.

Theorem 4.2 Let $n>0$ be a fixed integer. Let $\mathbf{C}^{0} \in \mathbf{H}$. There exists a solution, $\mathbf{C}_{n}$, to the problem:

find $\mathbf{C} \in W\left(\mathbf{H}^{1}\right)$ such that,

$$
\forall \phi \in \mathbf{H}^{1}, \quad\left(\frac{d \mathbf{C}}{d t}, \phi\right)+a(t, \mathbf{C}, \phi)=\left(\mathbf{G}_{n} \mathbf{C}, \phi\right),
$$

in the $\mathcal{D}^{\prime}(] 0, T[)$ sens, and $\mathbf{C}(0)=\mathbf{C}^{0}$.

Proof. From lemma 4.6, 4.7, 4.8 and the Schauder fixed-point theorem, the operator $\Theta$ has a fixed point, which is the solution sought $\square$.

\subsection{Step 2: letting $n \rightarrow \infty$}

We now pass to the limit as $n \rightarrow \infty$, in the equations,

$$
\begin{aligned}
& \forall \phi \in \mathbf{H}^{1}, \quad\left(\frac{d \mathbf{C}_{n}}{d t}(t), \phi\right)+a\left(t, \mathbf{C}_{n}, \phi\right)=\left(\mathbf{G}_{n} \mathbf{C}_{n}(t), \phi\right), \\
& \mathbf{C}_{n}(0)=\mathbf{C}^{0} .
\end{aligned}
$$

This is achieved in two steps: 
a) a priori estimations on the sequence $\mathbf{C}_{n}$,

b) extraction of subsequences and letting $n \rightarrow \infty$.

a) estimations. Let us show that:

(a.1) the sequence $\left(\mathbf{C}_{n}\right)_{n>0}$ is bounded in $L^{\infty}(0, T, \mathbf{H})$,

(a.2) the sequence $\left(\mathbf{C}_{n}\right)_{n>0}$ is bounded in $L^{2}\left(0, T, \mathbf{H}^{1}\right)$,

(a.3) the sequence $\left(\frac{d \mathbf{C}_{n}}{d t}\right)_{n>0}$ is bounded in $L^{2}\left(0, T,\left(\mathbf{H}^{1}\right)^{\prime}\right)$.

Taking, $\mathbf{C}_{n}$ as a test function in (4.4) we obtain,

$$
\frac{1}{2} \frac{d}{d t}\left\|\mathbf{C}_{n}\right\|^{2}+a\left(t, \mathbf{C}_{n}, \mathbf{C}_{n}\right)=\left(\mathbf{G}_{n} \mathbf{C}_{n}, \mathbf{C}_{n}\right),
$$

or,

$$
\frac{1}{2} \frac{d}{d t}\left\|\mathbf{C}_{n}\right\|^{2}+c_{0}\left\|\mathbf{C}_{n}\right\|_{1}^{2} \leq\left\|\mathbf{G}_{n} \mathbf{C}_{n}\right\|\left\|\mid \mathbf{C}_{n}\right\| \leq M_{g}\left\|\mathbf{C}_{n}\right\|^{2},
$$

and integrating on $[0, t]$, we obtain

$$
\left\|\mathbf{C}_{n}\right\|^{2}+2 c_{0} \int_{0}^{t}\left\|\mathbf{C}_{n}\right\|_{1}^{2} d s \leq 2 M_{g} \int_{0}^{t}\left\|\mathbf{C}_{n}\right\|^{2} d s+\left\|\mathbf{C}^{0}\right\|^{2} .
$$

Equation (4.5) gives

$$
\left\|\mathbf{C}_{n}\right\|^{2} \leq 2 M_{g} \int_{0}^{t}\left\|\mathbf{C}_{n}\right\|^{2} d s+\left\|\mathbf{C}^{0}\right\|^{2}
$$

Using Gronwall lemma, we have

$$
\left\|\mathbf{C}_{n}(t)\right\|^{2} \leq\left\|\mathbf{C}^{0}\right\|^{2} \exp \left(2 M_{g} T\right)
$$

and the sequence $\left(\mathbf{C}_{n}\right)_{n>0}$ is bounded in $L^{\infty}(0, T, \mathbf{H})$.

Equation (4.5) also gives

$$
\int_{0}^{t}\left\|\mathbf{C}_{n}\right\|_{1}^{2} d s \leq \frac{M_{g}}{c_{0}} \int_{0}^{t}\left\|\mathbf{C}_{n}\right\|^{2} d s+\frac{1}{2 c_{0}}\left\|\mathbf{C}^{0}\right\|^{2}
$$

and with (4.6)

$$
\int_{0}^{t}\left\|\mathbf{C}_{n}\right\|_{1}^{2} d s \leq \frac{M_{g} T}{c_{0}}\left\|\mathbf{C}^{0}\right\|^{2} \exp \left(2 M_{g} T\right)+\frac{1}{2 c_{0}}\left\|\mathbf{C}^{0}\right\|^{2} .
$$

Therefore the sequence $\left(\mathbf{C}_{n}\right)_{n>0}$ is bounded in $L^{2}\left(0, T, \mathbf{H}^{1}\right)$. 
Let us now give an estimation for the sequence, $\left(\frac{d \mathbf{C}_{n}}{d t}\right)_{n>0}$. We have

$$
\left|\left(\frac{d \mathbf{C}_{n}}{d t}, \phi\right)\right| \leq\left|a\left(t, \mathbf{C}_{n}, \phi\right)\right|+\left|\left(\mathbf{G}_{n} \mathbf{C}_{n}, \phi\right)\right|
$$

and therefore

$$
\left|\left(\frac{d \mathbf{C}_{n}}{d t}, \phi\right)\right| \leq M_{a}\left\|\mathbf{C}_{n}\right\|_{1}\|\phi\|_{1}+M_{g}\left\|\mathbf{C}_{n}\right\|_{1}\|\phi\|_{1},
$$

that is to say

$$
\begin{aligned}
& \int_{0}^{T}\left\|\frac{d \mathbf{C}_{n}}{d t}\right\|_{\left(\mathbf{H}^{1}\right)^{\prime}}^{2} \leq 2\left(M_{a}^{2}+M_{g}^{2}\right) \int_{0}^{T}\left\|\mathbf{C}_{n}\right\|_{1}^{2} \\
& \leq 2\left(M_{a}^{2}+M_{g}^{2}\right)\left(\frac{M_{g} T}{c_{0}}\left\|\mathbf{C}^{0}\right\|^{2} \exp \left(2 M_{g} T\right)+\frac{1}{2 c_{0}}\left\|\mathbf{C}^{0}\right\|^{2}\right),
\end{aligned}
$$

and the sequence $\left(\frac{d \mathbf{C}_{n}}{d t}\right)_{n>0}$ is bounded in $L^{2}\left(0, T,\left(\mathbf{H}^{1}\right)^{\prime}\right)$.

b) passing to the limit. Let us first recall that $\mathcal{D}(] 0, T\left[, \mathbf{H}^{1}\right) \subset W\left(\mathbf{H}^{1}\right)$. Therefore $\forall \phi \in \mathbf{H}^{1}$ and $\forall \varphi \in \mathcal{D}(] 0, T[)$, we have $\psi=\phi \otimes \varphi \in L^{2}\left(0, T, \mathbf{H}^{1}\right)$ and $\frac{d \psi}{d t} \in L^{2}\left(0, T,\left(\mathbf{H}^{1}\right)^{\prime}\right)$.

b.1) The term $\left(\frac{d \mathbf{C}_{n}}{d t}, \phi\right)$ : from (a.3), we are able to extract from the sequence $\left(\frac{d \mathbf{C}_{n}}{d t}\right)_{n>0}$ a subsequence (denoted in the same way) converging to some $\mathbf{h}$ in $L^{2}\left(0, T,\left(\mathbf{H}^{1}\right)^{\prime}\right)$ weak star, that is to say, for all $\phi \in \mathbf{H}^{1}$ and all $\varphi \in \mathcal{D}(] 0, T[)$,

$$
\lim _{n \rightarrow \infty} \int_{0}^{T}\left(\frac{d \mathbf{C}_{n}}{d t}, \phi\right) \varphi d s=\int_{0}^{T}(\mathbf{h}, \phi) \varphi d s .
$$

Moreover, by definition, we obtain

$$
\int_{0}^{T}\left(\frac{d \mathbf{C}_{n}}{d t}, \phi\right) \varphi d s=-\int_{0}^{T}\left(\mathbf{C}_{n}, \phi\right) \frac{d \varphi}{d t} d s
$$

From (a.2), we are able to extract from the sequence $\left(\mathbf{C}_{n}\right)_{n>0}$ a subsequence (denoted in the same way) converging to some $\mathbf{C}$ in $L^{2}\left(0, T, \mathbf{H}^{1}\right)$ weak. Therefore, for all $\phi \in \mathbf{H}^{1}$ and all $\varphi \in \mathcal{D}(] 0, T[)$,

$$
\lim _{n \rightarrow \infty}-\int_{0}^{T}\left(\mathbf{C}_{n}, \phi\right) \frac{d \varphi}{d t} d s=-\int_{0}^{T}(\mathbf{C}, \phi) \frac{d \varphi}{d t} d s,
$$

and $\mathbf{h}=\frac{d \mathbf{C}}{d t}$ in $L^{2}\left(0, T,\left(\mathbf{H}^{1}\right)^{\prime}\right)$. 
b.2) The term $a\left(t, \mathbf{C}_{n}, \phi\right)$ : from (b.1), we can suppose that the sequence $\left(\mathbf{C}_{n}\right)_{n>0}$ converges to $\mathbf{C}$ in $L^{2}\left(0, T, \mathbf{H}^{1}\right)$ weak. Therefore the sequence $\left(\partial_{x} \mathbf{C}_{n}\right)_{n>0}$ converges to $\partial_{x} \mathbf{C}$ in $L^{2}(0, T, \mathbf{H})$ weak. Then, for all $\phi \in \mathbf{H}^{1}$ and all $\varphi \in \mathcal{D}(] 0, T[)$,

$$
\lim _{n \rightarrow \infty} \int_{0}^{T} a\left(s, \mathbf{C}_{n}, \phi\right) \varphi d s=\int_{0}^{T} a(s, \mathbf{C}, \phi) \varphi d s .
$$

b.3) The term $\left(\mathbf{G}_{n} \mathbf{C}_{n}, \phi\right)$ : from (a.2), (a.3), and from the compacity of the injection $W\left(\mathbf{H}^{1}\right) \rightarrow L^{2}(0, T, \mathbf{H})$, we can suppose that the sequence $\left(\mathbf{C}_{n}\right)_{n>0}$ converges to $\mathbf{C}$ in $L^{2}(0, T, \mathbf{H})$ strong. Therefore, each $\mathbf{C}_{n, i}$, $i=1, \ldots 4$, converges to $\mathbf{C}_{i}$ in $L^{2}\left(0, T, L^{2}(0, L)\right)$ strong. From the inverse Lebesgue theorem (Brézis, ${ }^{5}$, theorem IV.9. page 58 ), we can suppose that:

(b.3.1) the sequences $\left(\mathbf{C}_{n, i}\right)_{n>0}, i=1, \ldots 4$, converge to $\mathbf{C}_{i}$ a.e. in $] 0, T[\times] 0, L[$.

(b.3.2) for $i=1$ to $4,\left|\mathbf{C}_{n, i}\right| \leq h_{i}, \forall n>0$, a.e. in $] 0, T[\times] 0, L\left[\right.$ and $h_{i} \in$ $L^{2}\left(0, T, L^{2}(0, L)\right)$.

As $\mathbf{g}_{n, i}(t, x, \mathbf{C})$ is continuous in its third variable, we deduce from (b.3.1) that $\forall \phi_{i} \in H^{1}(0, L)$ and $\forall \varphi \in \mathcal{D}(] 0, T[)$,

$$
\begin{aligned}
& u_{n, i}(t, x)=\mathbf{g}_{n, i}\left(t, x, \mathbf{C}_{n}(t, x)\right) \phi_{i}(x) \varphi(t) \\
& \rightarrow \underset{\mathbf{g}_{i}(t, x, \mathbf{C}(t, x)) \phi_{i}(x) \varphi(t),}{ } \rightarrow \infty
\end{aligned}
$$

a.e. in $] 0, T[\times] 0, L[$.

Moreover, from lemma 3.3 and with (b.3.2) we have,

$$
\left|u_{n, i}\right| \leq M_{i}\left(\sum_{i=1}^{4} h_{i}\right)\left|\phi_{i}\right||\varphi| \in L^{1}(] 0, T[\times] 0, L[),
$$

where the $M_{i}$ are constants. Thus, from the Lebesgue theorem on dominated convergence, we obtain,

$$
\lim _{n \rightarrow \infty} \int_{0}^{T} \int_{0}^{L} u_{n, i}(t, x) d x d t=\int_{0}^{T} \int_{0}^{L} \mathbf{g}_{i}(t, x, \mathbf{C}(t, x)) \phi_{i}(x) \varphi(t) d x d t,
$$

and finally, for all $\phi \in \mathbf{H}^{1}$ and all $\varphi \in \mathcal{D}(] 0, T[)$,

$$
\lim _{n \rightarrow \infty} \int_{0}^{T}\left(\mathbf{G}_{n} \mathbf{C}_{n}, \phi\right) \varphi=\int_{0}^{T}(\mathbf{G C}, \phi) \varphi .
$$

This concludes the proof of the existence of weak solutions to the one-dimensional NPZD-model.

\section{Positivity}


In this section we prove the second part of Theorem 3.1: if initial conditions $N^{0}, P^{0}, Z^{0}$ and $D^{0}$ are positive then solutions to the one-dimensional NPZD-model are positive for a.e. $t \in[0, T]$. To prove this, we need to treat each of the four equations seperately, in detail, and in a convenient order. We first show that $Z$ and $P$ are positive. Next we show that $D$ is positive using the fact that $Z$ and $P$ are positive. Finally, as $Z, P$ and $D$ are positive we obtain the positivity of $N$.

- Let us recall that for all $\mathbf{C} \in \mathbf{H}^{1}$ and all $t \in[0, T], a_{N}(t, N, N) \geq 0$, $a_{P}(t, P, P) \geq 0, a_{Z}(t, Z, Z) \geq 0$ and $a_{Z}(t, Z, Z) \geq 0$.

- $Z$ is positive:

Let $\mathbf{C}$ be a weak solution to the NPZD-model. Let us take

$$
-Z^{-}=-\max (0,-Z)
$$

as a test function. Since,

$$
\int_{0}^{L} \frac{\partial Z(t, x)}{\partial t} Z^{-}(t, x) d x=-\frac{1}{2} \frac{d}{d t}\left\|Z^{-}(t)\right\|_{L^{2}(0, L)}^{2}
$$

and

$$
a_{Z}\left(t, Z(t),-Z^{-}(t)\right)=a_{Z}\left(t, Z(t)^{-}, Z(t)^{-}\right),
$$

we obtain

$$
\frac{1}{2} \frac{d}{d t}\left\|Z(t)^{-}\right\|_{L^{2}(0, L)}^{2}+a_{Z}\left(t, Z(t)^{-}, Z(t)^{-}\right)=-\left(g_{Z}(\mathbf{C}(t)), Z(t)^{-}\right) .
$$

Let us detail the term $\left(g_{Z}(\mathbf{C}), Z^{-}\right)$.

$$
\begin{aligned}
\left(g_{Z}(\mathbf{C}), Z^{-}\right)_{L^{2}(0, L)}= & \int_{0}^{L}\left(a_{p} \frac{g_{z} P^{2}}{k_{z}+P^{2}} Z Z^{-}+a_{d} \frac{g_{z} D^{2}}{k_{z}+D^{2}} Z Z^{-}-m_{z} Z Z^{-}\right. \\
& \left.-\mu_{z} Z Z^{-}\right) \mathbb{1}_{] 0, l]}+\left(-\tau Z Z^{-}\right) \mathbb{1}_{l l, L[}+\lambda Z Z^{-} .
\end{aligned}
$$

As $Z Z^{-}=-\left(Z^{-}\right)^{2}$, we have

$$
\begin{aligned}
\left(g_{Z}(\mathbf{C}), Z^{-}\right)_{L^{2}(0, L)}= & \int_{0}^{L}\left(-\left(a_{p} \frac{g_{z} P^{2}}{k_{z}+P^{2}}\right)\left(Z^{-}\right)^{2}-\left(a_{d} \frac{g_{z} D^{2}}{k_{z}+D^{2}}\right)\left(Z^{-}\right)^{2}+m_{z}\left(Z^{-}\right)^{2}\right. \\
& \left.+\mu_{z}\left(Z^{-}\right)^{2}\right) \mathbb{1}_{] 0, l]}+\left(\tau\left(Z^{-}\right)^{2}\right) \mathbb{1}_{] l, L[}-\lambda\left(Z^{-}\right)^{2}
\end{aligned}
$$

and

$$
\begin{aligned}
\left(g_{Z}(\mathbf{C}), Z^{-}\right)_{L^{2}(0, L)} \geq & \int_{0}^{L}\left(-\left(a_{p} \frac{g_{z} P^{2}}{k_{z}+P^{2}}\right)\left(Z^{-}\right)^{2}\right. \\
& \left.-\left(a_{d} \frac{g_{z} D^{2}}{k_{z}+D^{2}}\right)\left(Z^{-}\right)^{2}\right) \mathbb{1}_{00, l]}-\lambda\left(Z^{-}\right)^{2}
\end{aligned}
$$


or

$$
\begin{aligned}
-\left(g_{Z}(\mathbf{C}), Z^{-}\right)_{L^{2}(0, L)} \leq & \int_{0}^{L}\left(\left(a_{p} \frac{g_{z} P^{2}}{k_{z}+P^{2}}\right)\left(Z^{-}\right)^{2}\right. \\
& \left.+\left(a_{d} \frac{g_{z} D^{2}}{k_{z}+D^{2}}\right)\left(Z^{-}\right)^{2}\right)+\lambda\left(Z^{-}\right)^{2}
\end{aligned}
$$

Thus

$$
\begin{aligned}
-\left(g_{Z}(\mathbf{C}), Z^{-}\right)_{L^{2}(0, L)} & \left.\leq \int_{0}^{L}\left(g_{z}\left(a_{p}+a_{d}\right)+\lambda\right)\left(Z^{-}\right)^{2}\right) \\
& =\left(g_{z}\left(a_{p}+a_{d}\right)+\lambda\right)\left\|Z^{-}\right\|_{L^{2}(0, L)}^{2}
\end{aligned}
$$

As $a_{Z}\left(t, Z^{-}, Z^{-}\right) \geq 0$, we obtain

$$
\frac{d}{d t}\left\|Z^{-}\right\|_{L^{2}(0, L)}^{2} \leq 2\left(g_{z}\left(a_{p}+a_{d}\right)+\lambda\right)\left\|Z^{-}\right\|_{L^{2}(0, L)}^{2} .
$$

Integrating this inequality on $[0, t]$ and using Gronwall's lemma, we obtain

$$
\left\|Z^{-}(t)\right\|_{L^{2}(0, L)}^{2} \leq\left\|Z^{-}(0)\right\|_{L^{2}(0, L)}^{2} \exp \left(2\left(g_{z}\left(a_{p}+a_{d}\right)+\lambda\right) t\right) .
$$

Therefore $Z$ is positive.

- $P$ is positive:

In the same manner, let us examine the term $\left(g_{P}(\mathbf{C}), P^{-}\right)_{L^{2}(0, L)}$.

$$
\begin{aligned}
\left(g_{P}(\mathbf{C}), P^{-}\right)_{L^{2}(0, L)} & =\int_{0}^{L}\left(\mu_{p}(1-\gamma) L_{I} L_{N} P P^{-}-\left(\frac{g_{z} P^{2}}{k_{z}+P^{2}} Z P^{-}\right)\right. \\
& \left.-m_{p} P P^{-}\right) \mathbb{1}_{] 0, l]}+\left(-\tau P P^{-}\right) \mathbb{1}_{l l, L[}+\lambda P P^{-} \\
& =\int_{0}^{L}\left(-\mu_{p}(1-\gamma) L_{I} L_{N}\left(P^{-}\right)^{2}-\left(\frac{g_{z} P^{2}}{k_{z}+P^{2}} Z P^{-}\right)\right. \\
& \left.+m_{p}\left(P^{-}\right)^{2}\right) \mathbb{1}_{] 0, l]}+\left(\tau\left(P^{-}\right)^{2}\right) \mathbb{1}_{] l, L[}-\lambda\left(P^{-}\right)^{2} \\
& \geq \int_{0}^{L}\left(-\mu_{p}(1-\gamma) L_{I} L_{N}\left(P^{-}\right)^{2}\right. \\
& \left.-\left(\frac{g_{z} P^{2}}{k_{z}+P^{2}} Z P^{-}\right)\right) \mathbb{1}_{] 0, l]}-\lambda\left(P^{-}\right)^{2}
\end{aligned}
$$

and

$$
\begin{aligned}
-\left(g_{P}(\mathbf{C}), P^{-}\right)_{L^{2}(0, L)} & \leq \int_{0}^{L} \mu_{p}(1-\gamma) L_{I} L_{N}\left(P^{-}\right)^{2} \\
& +\left(\frac{-g_{z} P}{k_{z}+P^{2}} Z\left(P^{-}\right)^{2}\right) \mathbb{1}_{] 0, l]}+\lambda\left(P^{-}\right)^{2}
\end{aligned}
$$

The function $x \mapsto \frac{-x}{k_{z}+x^{2}}$ is bounded by $\frac{1}{2 \sqrt{k_{z}}}$ on $\mathbb{R}$.

$L_{N}$ and $L_{I}$ are bounded by 1 . 
$Z(t)$, is a solution to the NPZD-model and therefore belongs to $H^{1}(0, L) \subset$ $L^{\infty}(0, L)$. Hence we have, $\forall t, Z(t) \leq\|Z(t)\|_{\infty}$, and

$$
-\left(g_{P}(\mathbf{C}), P^{-}\right)_{L^{2}(0, L)} \leq\left(\lambda+\mu_{p}(1-\gamma)+g_{z} \frac{1}{2 \sqrt{k_{z}}}\|Z(t)\|_{\infty}\right)\left\|P^{-}(t)\right\|_{L^{2}(0, L)}^{2} .
$$

We conclude in the same way to obtain

$$
\begin{aligned}
\left\|P^{-}(t)\right\|_{L^{2}(0, L)}^{2} & \leq\left\|P^{-}(0)\right\|_{L^{2}(0, L)}^{2} \exp \left(\int _ { 0 } ^ { t } 2 \left(\lambda+\mu_{p}(1-\gamma)\right.\right. \\
& \left.\left.+g_{z} \frac{1}{2 \sqrt{k_{z}}}\|Z(s)\|_{\infty}\right) d s\right) .
\end{aligned}
$$

- $D$ is positive:

$$
\begin{aligned}
\left(g_{D}(\mathbf{C}), D^{-}\right)_{L^{2}(0, L)} & =\int_{0}^{L}\left(\left(1-a_{p}\right)\left(\frac{g_{z} P^{2}}{k_{z}+P^{2}} Z D^{-}\right)-a_{d}\left(\frac{g_{z} D^{2}}{k_{z}+D^{2}} Z D^{-}\right)\right. \\
& \left.+m_{p} P D^{-}+m_{z} Z D^{-}+\mu_{d}\left(D^{-}\right)^{2}\right) \mathbb{1}_{] 0, l]} \\
& +\left(\tau\left(D^{-}\right)^{2}\right) \mathbb{1}_{l l, L[}+\lambda D D^{-} .
\end{aligned}
$$

Because $P, Z$ and $D^{-}$are positive, we obtain

$$
\begin{aligned}
\left(g_{D}(\mathbf{C}), D^{-}\right)_{L^{2}(0, L)} & \geq \int_{0}^{L}\left(-a_{d}\left(\frac{g_{z} D^{2}}{k_{z}+D^{2}} Z D^{-}\right)\right) \mathbb{1}_{00, l]}-\lambda\left(D^{-}\right)^{2}, \\
-\left(g_{D}(\mathbf{C}), D^{-}\right)_{L^{2}(0, L)} & \leq \int_{0}^{L}\left(-a_{d}\left(\frac{g_{z} D}{k_{z}+D^{2}} Z\left(D^{-}\right)^{2}\right)\right) \mathbb{1}_{] 0, l]}+\lambda\left(D^{-}\right)^{2}, \\
-\left(g_{D}(\mathbf{C}), D^{-}\right)_{L^{2}(0, L)} & \leq \int_{0}^{L}\left(a_{d}\left(g_{z} \frac{1}{2 \sqrt{k_{z}}}\|Z\|_{\infty}\right)\left(D^{-}\right)^{2}\right) \mathbb{1}_{] 0, l]}+\lambda\left(D^{-}\right)^{2}, \\
-\left(g_{D}(\mathbf{C}), D^{-}\right)_{L^{2}(0, L)} & \leq\left(\lambda+a_{d}\left(g_{z} \frac{1}{2 \sqrt{k_{z}}}\|Z\|_{\infty}\right)\right)\left\|D^{-}\right\|_{L^{2}(0, L)}^{2} .
\end{aligned}
$$

Hence

$$
\frac{d}{d t}\left\|D^{-}\right\|_{L^{2}(0, L)}^{2} \leq 2\left(\lambda+a_{d}\left(g_{z} \frac{1}{2 \sqrt{k_{z}}}\|Z\|_{\infty}\right)\right)\left\|D^{-}\right\|_{L^{2}(0, L)}^{2},
$$

and we can conclude.

- $N$ is positive:

$$
\begin{aligned}
\left(g_{N}(\mathbf{C}), N^{-}\right)_{L^{2}(0, L)} & =\int_{0}^{L}\left(-\mu_{p}(1-\gamma) L_{I} L_{N} P N^{-}+\mu_{z} Z N^{-}\right. \\
& \left.+\mu_{d} D N^{-}\right) \mathbb{1}_{] 0, l]}+\left(\tau(P+Z+D) N^{-}\right) \mathbb{1}_{l, L[}+\lambda N N^{-} .
\end{aligned}
$$

Because $P, Z, D$ and $N^{-}$are positive, we have

$$
\begin{aligned}
& \left(g_{N}(\mathbf{C}), N^{-}\right)_{L^{2}(0, L)} \geq \int_{0}^{L}\left(-\mu_{p}(1-\gamma) L_{I} L_{N} P N^{-}\right) \mathbb{1}_{] 0, l]}-\lambda\left(N^{-}\right)^{2}, \\
& -\left(g_{N}(\mathbf{C}), N^{-}\right)_{L^{2}(0, L)} \leq \int_{0}^{L}\left(-\mu_{p}(1-\gamma) L_{I} \frac{1}{k_{n}+|N|} P\left(N^{-}\right)^{2}\right) \mathbb{1}_{00, l]}+\lambda\left(N^{-}\right)^{2} .
\end{aligned}
$$


Once again we can conclude and the proof of theorem 3.1 is complete.

Hence, if initial concentrations are positive then concentrations are always positive and both models, with or without absolute values in the nonlinear terms, are equivalent.

\section{Existence, positivity and uniqueness for different $G_{P}, G_{D}, L_{I}$ and zooplankton mortality formulations}

Functions used to parameterize biological fluxes such as zooplankton grazing on phytoplankton, $G_{P}$, or on detritus, $G_{D}$, light limited growth rate, $L_{I}$ or zooplankton mortality (which is a constant, $m_{z}$, in our model), vary from one modeling study to another. One can wonder if the existence result still applies with these different formulations. To answer this question it should be noticed that the key argument used in the proof is the fact that the nonlinear reaction terms allow us to define a nonlinear continuous operator $\mathbf{G}$ satisfying $\|\mathbf{G C}\|_{L^{2}(0, T, \mathbf{H})} \leq M_{g}\|\mathbf{C}\|_{L^{2}(0, T, \mathbf{H})}$. Therefore, as all the functions listed in Table 2, found in the literature, are continuous and bounded on $\mathbb{R}^{+}$or $\left(\mathbb{R}^{+}\right)^{2}$, the existence result stays correct. Positivity can also easily be checked for all these different formulations. It should however be mentioned that some studies use a quadratic zooplankton mortality term which can not be treated with the method we propose.

Let us now concentrate on the question of the uniqueness of weak solutions to the one-dimensional NPZD-model.

In order to prove uniqueness we need the nonlinear reaction terms to satisfy a local Lipschitz condition which was not needed to obtain the existence result. To verify that such a condition holds we examine in some details the optical model from which the $P A R(t, x, P)$ term and consequently the $L_{I}(t, x, P)$ term are calculated. In the different equations $L_{I}(t, x, P)$ allways appears in the product form $P L_{I}(t, x, P)$. Concerning this product the desired local Lipschitz condition reads as follows:

for all $(t, x) \in[0, T] \times[0, L]$, and all $P, \hat{P} \in[0,+\infty[$,

$$
\left|P L_{I}(t, x, P)-\hat{P} L_{I}(t, x, \hat{P})\right| \leq K_{I}(P, \hat{P})|P-\hat{P}|,
$$

where $K_{I}$ is a continuous nonnegative real-valued function which is increasing in each variable.

In the optical model we considered, two different wavelengths are taken into account and the absorption coefficients depend on the local phytoplankton concentrations:

$$
\begin{aligned}
P A R(t, x, P)= & Q(t)\left(\exp \left(-\left(k_{g o}+k_{g p}\left(\frac{12 P r_{d}}{r_{p g} r_{c}}\right)^{l_{g}}\right) x\right)\right. \\
& \left.+\exp \left(-\left(k_{r o}+k_{r p}\left(\frac{12 P r_{d}}{r_{p g} r_{c}}\right)^{l_{r}}\right) x\right)\right) .
\end{aligned}
$$

$Q(t)$ is proportional to the irradiance intensity hitting the sea surface at time $t$. Parameters are given in Table 3. Let us suppose that $Q(t) \in L^{\infty}(0, T)$ and that 
Table 2: Different parameterizations found in the literature. All parameters are positive constants.

\begin{tabular}{|c|c|c|}
\hline \multirow[t]{3}{*}{$\begin{array}{l}Z \text { grazing on } \\
P, G_{P}\end{array}$} & $\frac{g_{z} P^{2}}{k_{z}+P^{2}}$ & $\begin{array}{l}\text { this study and e.g. } \\
\text { Fennel et al. }{ }^{13}\end{array}$ \\
\hline & $\frac{g_{z} P^{2}}{k_{z}+P^{2}+D^{2}}$ & e.g. Losa et al. ${ }^{21}$ \\
\hline & $\frac{g_{z} r P^{2}}{k_{z}(r P+(1-r) D)+r P^{2}+(1-r) D^{2}}$ & $\begin{array}{l}\text { e.g. Fasham et al. } \\
10\end{array}$ \\
\hline \multirow[t]{3}{*}{$\begin{array}{l}Z \text { grazing on } \\
D, G_{D}\end{array}$} & $\frac{g_{z} D^{2}}{k_{z}+D^{2}}$ & $\begin{array}{l}\text { this study and e.g. } \\
\text { Fennel et al. } 13\end{array}$ \\
\hline & $\frac{g_{z} D^{2}}{k_{z}+P^{2}+D^{2}}$ & e.g. Losa et al. ${ }^{21}$ \\
\hline & $\frac{g_{z} r D^{2}}{k_{z}(r P+(1-r) D)+r P^{2}+(1-r) D^{2}}$ & $\begin{array}{l}\text { e.g. Fasham et al. } \\
10\end{array}$ \\
\hline \multirow{2}{*}{$\begin{array}{l}\text { light limited } \\
\text { growth rate, } \\
L_{I}\end{array}$} & $1-\exp \left(-P A R(t, x, P) / k_{p a r}\right)$ & $\begin{array}{l}\text { this study and e.g. } \\
\text { Lévy et al. } 16\end{array}$ \\
\hline & $\frac{v_{p} \alpha P A R(t, x, P)}{\left(v_{p}^{2}+\alpha^{2} P A R(t, x, P)^{2}\right)^{1 / 2}}$ & e.g. Spitz et al. ${ }^{26}$ \\
\hline \multirow[t]{2}{*}{ Z mortality } & $m_{z}$ & $\begin{array}{l}\text { this study and e.g. } \\
\text { Lévy et al. }{ }^{17}\end{array}$ \\
\hline & $\frac{m_{z} Z}{k+Z}$ & e.g. Losa et al. ${ }^{21}$ \\
\hline
\end{tabular}


$Q(t) \geq 0$. Even though the exponents $l_{g}$ and $l_{r}$ satisfy $0<l_{g}<1$ and $0<l_{r}<1$, an easy calculation of the derivative, $\frac{d}{d P}\left(P L_{I}(t, x, P)\right)$, shows that with such an optical model property (6.7) is satisfied with:

$K_{I}(P, \hat{P})=1+\frac{\|Q\|_{\infty} L}{k_{p a r}}\left(k_{g p} l_{g}\left(\frac{12 r_{d}}{r_{p g} r_{c}}\right)^{l_{g}}(\max (P, \hat{P}))^{l_{g}}+k_{r p} l_{r}\left(\frac{12 r_{d}}{r_{p g} r_{c}}\right)^{l_{r}}(\max (P, \hat{P}))^{l_{r}}\right)$.

In the literature $P A R(t, x, P)$ is often parameterized by,

$$
P A R(t, x, P)=Q(t) \exp \left(-\left(k_{1}+k_{2} P\right) x\right)
$$

where $k_{1}$ and $k_{2}$ are positive constants. With this simpler formulation property (6.7) is clearly satisfied.

The following two lemmas give the local Lipschitz property satisfied by all four reaction terms of the NPZD-model.

Lemma 6.9 The nonlinear reaction terms $g_{N}, g_{P}, g_{Z}$ and $g_{D}$ satisfy: for all $(t, x) \in[0, T] \times[0, L]$, and all $\mathbf{C}, \hat{\mathbf{C}} \in\left(\mathbb{R}^{+}\right)^{4}$,

$$
\begin{aligned}
& \left|g_{N}(t, x, \mathbf{C})-g_{N}(t, x, \hat{\mathbf{C}})\right| \leq K_{N}(P, \hat{P})(|N-\hat{N}|+|P-\hat{P}|+|Z-\hat{Z}|+|D-\hat{D}|), \\
& \left|g_{P}(t, x, \mathbf{C})-g_{P}(t, x, \hat{\mathbf{C}})\right| \leq K_{P}(P, \hat{P})(|N-\hat{N}|+|P-\hat{P}|+|Z-\hat{Z}|+|D-\hat{D}|), \\
& \left|g_{Z}(t, x, \mathbf{C})-g_{Z}(t, x, \hat{\mathbf{C}})\right| \leq K_{Z}(Z, \hat{Z})(|N-\hat{N}|+|P-\hat{P}|+|Z-\hat{Z}|+|D-\hat{D}|), \\
& \left|g_{D}(t, x, \mathbf{C})-g_{D}(t, x, \hat{\mathbf{C}})\right| \leq K_{D}(Z, \hat{Z})(|N-\hat{N}|+|P-\hat{P}|+|Z-\hat{Z}|+|D-\hat{D}|),
\end{aligned}
$$

where $K_{N}, K_{P}, K_{Z}, K_{D}$ are continuous nonnegative real-valued functions which are increasing in each variable.

Proof. Functions $l(x)=\frac{x}{k_{n}+x}$ and $g(x)=\frac{x^{2}}{k_{z}^{2}+x^{2}}$ are continuously differentiable on $[0,+\infty[$, and

$$
\left|l^{\prime}(x)\right| \leq \frac{1}{k_{n}}, \quad\left|g^{\prime}(x)\right| \leq \frac{3 \sqrt{3}}{8 \sqrt{k_{z}}}
$$

Therefore $l$ and $g$ are Lipschitz continuous.

It is clear that

$$
\begin{aligned}
\left|g_{N}(t, x, \mathbf{C})-g_{N}(t, x, \hat{\mathbf{C}})\right| & \leq \mu_{p}(1-\gamma)\left|L_{I}(t, x, P) P L_{N}(N)-L_{I}(t, x, \hat{P}) \hat{P} L_{N}(\hat{N})\right| \\
& +\left(\mu_{z}+\tau\right)|Z-\hat{Z}|+\left(\mu_{d}+\tau\right)|D-\hat{D}| \\
& +\tau|P-\hat{P}|+\lambda|N-\hat{N}| .
\end{aligned}
$$

Now since

$$
\begin{aligned}
\left|L_{I}(t, x, P) P L_{N}(N)-L_{I}(t, x, \hat{P}) \hat{P} L_{N}(\hat{N})\right|= & \mid L_{I}(t, x, P) P\left(L_{N}(N)-L_{N}(\hat{N})\right) \\
& +L_{N}(\hat{N})\left(L_{I}(t, x, P) P\right)-L_{I}(t, x, \hat{P}) \hat{P} \mid
\end{aligned}
$$

we have

$$
\left|L_{I}(t, x, P) P L_{N}(N)-L_{I}(t, x, \hat{P}) \hat{P} L_{N}(\hat{N})\right| \leq \frac{1}{k_{n}} \max (P, \hat{P})|N-\hat{N}|+K_{I}(P, \hat{P})|P-\hat{P}| .
$$


We then define

$K_{N}(P, \hat{P})=\max \left(\lambda+\mu_{p}(1-\gamma) \frac{1}{k_{n}} \max (P, \hat{P}), \tau+\mu_{p}(1-\gamma) K_{I}(P, \hat{P}), \mu_{z}+\tau, \mu_{d}+\tau\right)$.

$K_{P}, K_{Z}$ and $K_{D}$ are obtained in the same way $\square$.

Lemma 6.10 For $t \in[0, T]$ and for positive $\mathbf{C}(t), \hat{\mathbf{C}}(t) \in \mathbf{H}^{1}$, there exists a constant $L_{\infty}$, depending on $\|\mathbf{C}(t)\|_{\infty}$ and $\|\hat{\mathbf{C}}(t)\|_{\infty}$, such that the operator, $\mathbf{G}$, satisfies,

$$
\|\mathbf{G C}(t)-\mathbf{G} \hat{\mathbf{C}}(t)\| \leq L_{\infty}\|\mathbf{C}(t)-\hat{\mathbf{C}}(t)\| .
$$

Proof. From lemma 3.1, $\mathbf{H}^{1} \subset \mathbf{L}^{\infty}$. From lemma 6.9 we have,

$$
\begin{aligned}
& \|\mathbf{G} \mathbf{C}(t)-\mathbf{G} \hat{\mathbf{C}}(t)\|^{2} \\
& =\int_{0}^{L}\left|g_{N}(t, x, \mathbf{C}(t, x))-g_{N}(t, x, \hat{\mathbf{C}}(t, x))\right|^{2}+\left|g_{P}(t, x, \mathbf{C}(t, x))-g_{P}(t, x, \hat{\mathbf{C}}(t, x))\right|^{2} \\
& +\left|g_{Z}(t, x, \mathbf{C}(t, x))-g_{Z}(t, x, \hat{\mathbf{C}}(t, x))\right|^{2}+\left|g_{D}(t, x, \mathbf{C}(t, x))-g_{D}(t, x, \hat{\mathbf{C}}(t, x))\right|^{2} d x \\
& \leq \operatorname{cte}\left(K_{N}\left(\|P(t)\|_{\infty},\|\hat{P}(t)\|_{\infty}\right)\right)^{2}\left[\|N(t)-\hat{N}(t)\|_{L^{2}(0, L)}^{2}\right. \\
& \left.+\|P(t)-\hat{P}(t)\|_{L^{2}(0, L)}^{2}+\|Z(t)-\hat{Z}(t)\|_{L^{2}(0, L)}^{2}+\|D(t)-\hat{D}(t)\|_{L^{2}(0, L)}^{2}\right] \\
& +\ldots \\
& \leq L_{\infty}\left(\|\mathbf{C}(t)\|_{\infty},\|\hat{\mathbf{C}}(t)\|_{\infty}\right)^{2}\|\mathbf{C}(t)-\hat{\mathbf{C}}(t)\|^{2}
\end{aligned}
$$

Elementary calculations show that functions of Table 2 are continuously differentiable on $\mathbb{R}^{+}$or $\left(\mathbb{R}^{+}\right)^{2}$, with bounded first derivatives. Therefore they are Lipschitz continuous and the uniqueness result presented below also holds for these formulations.

Proposition 6.4 The weak solution to the one-dimensional NPZD-model, $\mathbf{C} \in$ $W\left(\mathbf{H}^{1}\right)$, is unique.

Proof. Let us suppose that there are two solutions $\mathbf{C}^{1}$ and $\mathbf{C}^{2} \in W\left(\mathbf{H}^{1}\right)$. They satisfy

$$
\forall \phi \in \mathbf{H}^{1}, \quad\left(\frac{d}{d t}\left(\mathbf{C}^{1}-\mathbf{C}^{2}\right), \phi\right)+a\left(t, \mathbf{C}^{1}-\mathbf{C}^{2}, \phi\right)=\left(\mathbf{G C}^{1}-\mathbf{G C}^{2}, \phi\right),
$$

Let us choose $\phi=\mathbf{C}^{1}-\mathbf{C}^{2}$ as a test function. Using the coerciveness of $a$ and the Cauchy-Schwarz inequality, we obtain

$$
\frac{1}{2} \frac{d}{d t}\left\|\mathbf{C}^{1}(t)-\mathbf{C}^{2}(t)\right\|^{2}+c_{0}\left\|\mathbf{C}^{1}(t)-\mathbf{C}^{2}(t)\right\|_{1}^{2} \leq\left\|\mathbf{G} \mathbf{C}^{1}(t)-\mathbf{G C}^{2}(t)\right\|\left\|\mathbf{C}^{1}(t)-\mathbf{C}^{2}(t)\right\| .
$$


With Young's inequality, we obtain

$$
\begin{aligned}
& \frac{1}{2} \frac{d}{d t}\left\|\mathbf{C}^{1}(t)-\mathbf{C}^{2}(t)\right\|^{2}+c_{0}\left\|\mathbf{C}^{1}(t)-\mathbf{C}^{2}(t)\right\|_{1}^{2} \\
& \leq \frac{1}{2 \alpha}\left\|\mathbf{G} \mathbf{C}^{1}(t)-\mathbf{G C}^{2}(t)\right\|^{2}+\frac{\alpha}{2}\left\|\mathbf{C}^{1}(t)-\mathbf{C}^{2}(t)\right\|^{2},
\end{aligned}
$$

with $\alpha=2 c_{0}$,

$$
\frac{d}{d t}\left\|\mathbf{C}^{1}(t)-\mathbf{C}^{2}(t)\right\|^{2} \leq \frac{1}{2 c_{0}}\left\|\mathbf{G} \mathbf{C}^{1}(t)-\mathbf{G C}^{2}(t)\right\|^{2} .
$$

From lemma (6.10) we obtain

$$
\frac{d}{d t}\left\|\mathbf{C}^{1}(t)-\mathbf{C}^{2}(t)\right\|^{2} \leq \frac{1}{2 c_{0}} L_{\infty}^{2}(t)\left\|\mathbf{C}^{1}(t)-\mathbf{C}^{2}(t)\right\|^{2} .
$$

Thus, integrating on $[0, t]$ and using Gronwall's lemma

$$
\left\|\mathbf{C}^{1}(t)-\mathbf{C}^{2}(t)\right\|^{2} \leq\left\|\mathbf{C}^{1}(0)-\mathbf{C}^{2}(0)\right\|^{2} \exp \left(\int_{0}^{t} \frac{1}{2 c_{0}} L_{\infty}^{2}(s) d s\right) .
$$

This concludes the proof $\square$.

Table 3: Optical model parameters

\begin{tabular}{|l|c|c|c|}
\hline parameter & name & value & unit \\
\hline Redfield ratio C:N & $r_{d}$ & 6.625 & \\
contribution of Chl to absorbing & $r_{p g}$ & 0.7 & \\
pigments & & & \\
carbone:chlorophyll ratio & $r_{c}$ & 55 & $m g C . m g C h l a^{-1}$ \\
water absorption in red & $k_{r o}$ & 0.225 & $m^{-1}$ \\
water absorption in green & $k_{g o}$ & 0.0232 & $m^{-1}$ \\
pigment absorption in red & $k_{r p}$ & 0.037 & $m^{-1} \cdot\left(\mathrm{mgChl.m}^{-3}\right)^{-l_{r}}$ \\
pigment absorption in green & $k_{g p}$ & 0.074 & $m^{-1} \cdot\left(m g C h l . m^{-3}\right)^{-l_{g}}$ \\
power law for absorption in red & $l_{r}$ & 0.629 & \\
power law for absorption in green & $l_{g}$ & 0.674 & \\
\hline
\end{tabular}

\section{Conclusion}

We have presented a qualitative analysis of a one-dimensional biological NPZDmodel. This model describes the evolution over time and space of four biological variables, phytoplankton, zooplankton, nutrients and detritus. The only physical process which is taken into account is vertical diffusion and the biological model is imbedded in a physical turbulence model which we did not give explicitly but appeared as a space and time-dependent mixing coefficient. The model's equation for detritus also contains an advection term which represents the sinking of detritus with a constant speed. All four variables interact through nonlinear reaction 
terms which depend on space and time through the action of light and present a discontinuity in the space variable at a particular depth.

We have formulated an initial-boundary value problem and proved existence of a unique weak solution to it. Furthermore, a detailed investigation of the reaction terms enabled us to prove positivity of the solution. This is biologically important since variables represent concentrations which should always be positive quantities. We have also shown that the result still holds if different parameterizations of biological processes found in the biogeochemical modeling literature are used.

The analysis conducted in this paper is a necessary first step towards the investigation of qualitative properties other than positivity which might be of interest. For example Boushaba et al. ${ }^{4}$ deal with the problem of determining the asymptotic behavior of solutions to their phytoplankton model. One could also wish to investigate the bifurcational structure of the NPZD-model even though the complexity of the analytical formulation of the equations might constitute a difficulty. This type of study could help the understanding of the modifications of evolution of the NPZD system under minor changes in the values of parameters reported in Edwards ${ }^{9}$ and Faugeras et al. ${ }^{12}$.

Eventually we would like to point out that the analysis we presented can easily be extended to models containing any number, $n$, of biological variables as long as the nonlinearities allow us to define a continuous nonlinear operator $\mathbf{G}$ satisfying $\|\mathbf{G C}\|_{L^{2}\left(0, T,\left(L^{2}(0, L)\right)^{n}\right)} \leq M_{g}\|\mathbf{C}\|_{L^{2}\left(0, T,\left(L^{2}(0, L)\right)^{n}\right)}$. However, in such more complex models, the question of positivity seems to be delicate as equations have to be treated one after the other, and the right order has to be found as the positivity of some variables can depend on the positivity of others. Let us also mention that the analysis can be extended to three-dimensional models in which not only mixing coefficients but also velocities, calculated by an ocean circulation model, are included in the system of partial differential equations constituing the biological model. These velocities can be included in the bilinear form, $a(t, . .$.$) , as v_{d}$, the detritus sedimentation speed, is in the formulation of the initial-boundary value problem we studied.

\section{Acknowledgements.}

The author is grateful to his thesis supervisors, Jacques Blum and Jacques Verron, for their guidance. The author also thanks Marina Lévy and Laurent Mémery for helpful discussions on physical and biological models, and Jean-Pierre Puel for his mathematical advices.

1. V. Andersen and L. Prieur, One-month study in the open NW Mediterranean Sea (DYNAPROC experiment, May 1995): overview of the hydrobiologeochemical structures and effects of wind events, Deep-Sea Research I 47 (2000) 397-422.

2. M. Artola, Existence and uniqueness for a diffusion system with coupling, in Recent advances in nonlinear elliptic and parabolic problems, Pitman Research Notes in mathematics Series 208, (1989), 112-138.

3. J.P. Aubin, Un théorème de compacité, C. R. Acad. Sci. Paris 256 (1963) 5042-5044.

4. K. Boushaba, O. Arino and A. Boussouar, A mathematical model for phytoplankton, Math. Models and Meth. Appl. Sci. 12(6) (2002) 871-901. 
5. H. Brézis, Analyse fonctionnelle. Théorie et applications, (Masson, 1992).

6. V. Carmillet, J.-M. Brankart, P. Brasseur, H. Drange, G. Evensen and J. Verron, A singular evolutive extended Kalman filter to assimilate ocean color data in a coupled physical-biochemical model of the North Atlantic ocean, Ocean Modelling 3 (2001) 167192.

7. R. Dautray and J.-L. Lions, Analyse mathématique et calcul numérique pour les sciences et les techniques, Vol. 8 (Masson, 1988).

8. S.C. Doney, D.M. Glover and R.G. Najjar, A new coupled, one dimensional biologicalphysical model for the upper ocean: applications to the JGOFS Bermuda Atlantic Timeseries Study (BATS) site., Deep-Sea Research II 43 (1996) 591-624.

9. A.M. Edwards, Adding detritus to a nutrient-phytoplankton-zooplankton model: a dynamical-systems approach, Journal of Plankton Research 23 (2001) 389-413.

10. M.J.R. Fasham, H.W. Ducklow and S.M. McKelvie, A nitrogen-based model of plankton dynamics in the oceanic mixed-layer, J. Mar. Res. 48 (1990) 591-639.

11. M.J.R. Fasham, J.L. Sarmiento, R.D. Slater, H.W. Ducklow and R. Williams, Ecosystem behavior at Bermuda Station "S" and Ocean Weather Station "India": a general circulation model and observational analysis, Global Biogeochem. Cycles 7 (1993) 379415 .

12. B. Faugeras, M. Lévy, L. Mémery, J. Verron, J. Blum and I. Charpentier, Can biogeochemical fluxes be recovered from nitrate and chlorophyll data? A case study assimilating data in the Northwestern Mediterranean Sea at the JGOFS-DYFAMED station., J. Mar. Sys. , 2002, to appear.

13. K. Fennel, M. Losch, J. Schröter and W. Manfred, Testing a marine ecosystem model: sensitivity analysis and parameter optimization, J. Mar. Sys. 28 (2001) 45-63.

14. P. Gaspar, Y. Gregories, J.M. Lefevre, A simple eddy kinetic energy model for simulations of the oceanic vertical mixing: tests at station Papa and long term upper ocean study site, Journal of Geophysical Research 95 (1990) 16179-16193.

15. M.A. Krasnosel'skii, Topological Methods in the Theory of Nonlinear Integral Equations, (Pergamon Press, 1964).

16. M. Lévy, L. Mémery and J.-M. André, Simulation of primary production and export fluxes in the Northwestern Mediterranean Sea, J. Mar. Res. 56 (1998) 197-238.

17. M. Lévy, L. Mémery and G. Madec, The onset of a bloom after deep winter convection in the Northwestern Mediterranean Sea : mesoscale process study with a primitive equation model, J. Mar. Sys. 16 (1998) 7-21.

18. R. Lewandosky, Analyse Mathématique et Océanographie, Recherche en mathématiques appliquées (Masson, 1997).

19. J.-L. Lions, Quelques méthodes de résolution de problèmes aux limites non linéaires (Gauthier Villard, 1969)

20. J.-L. Lions and E. Magenes, Problèmes aux limites non homogènes et applications Vol. 1 (Dunod, 1968).

21. N.L. Losa, G.A. Kivman and V.A. Ryabchenko, Weak constraint parameter estimation for a simple ocean ecosystem model: what can we learn about the model and data?, J. Mar. Sys., 2002, submitted.

22. L. Mémery, M. Lévy, S. Vérant and L. Mervilat, The relevant time scales in estimating the air - sea $\mathrm{CO}_{2}$ exchange in a mid latitude region, Deep-Sea Research II 49 (2002) 2067-2092.

23. J.R. Moisan, E.E. Hofmann and D.B. Haidvogel, Modeling nutrient and plankton processes in the California coastal transition zone. 2. A three-dimensional physical-biooptical model., Journal of Geophysical Research 101 (1996) 22677-22691.

24. A. Pazy, Semigroups of Linear Operators and Applications to Partial Dif- 
ferential Equations, Applied Mathematical Sciences (Springer-Verlag, 1983).

25. P. Prunet, J.F. Minster, D. Ruiz-Pino and I. Dadou, Assimilation of surface data in a one-dimensional physical-biogeochemical model of the surface ocean: 1. Method and preliminary results, Global Biogeochem. Cycles 10 (1996) 111-138.

26. Y.H. Spitz, J.R. Moisan, M.R. Abbot and J.G. Richman, Data assimilation and a pelagic ecosystem model: parameterization using time series observations, J. Mar. Sys. 16 (1998) 51-68.

27. J.H. Steele and E.W. Henderson, The role of predation in plankton models., Journal of Plankton Research 14 (1992) 157-172.

28. A.H. Taylor, A.J. Watson, J.E. Ainsworth, J.E. Robertson and D.R. Turner, A modelling investigation of the role of phytoplankton in the balance of carbon at the surface of the North Atlantic, Global Biogeochem. Cycles 5 (1991) 151-171. 